\title{
Efficacy of interventions that use apps to improve diet, physical activity and sedentary behaviour: a systematic review
}

Stephanie Schoeppe ${ }^{1 *}$, Stephanie Alley ${ }^{1}$, Wendy Van Lippevelde ${ }^{2}$, Nicola A. Bray ${ }^{1}$, Susan L. Williams ${ }^{3}$, Mitch J. Duncan ${ }^{4}$ and Corneel Vandelanotte ${ }^{1}$

\begin{abstract}
Background: Health and fitness applications (apps) have gained popularity in interventions to improve diet, physical activity and sedentary behaviours but their efficacy is unclear. This systematic review examined the efficacy of interventions that use apps to improve diet, physical activity and sedentary behaviour in children and adults.

Methods: Systematic literature searches were conducted in five databases to identify papers published between 2006 and 2016. Studies were included if they used a smartphone app in an intervention to improve diet, physical activity and/or sedentary behaviour for prevention. Interventions could be stand-alone interventions using an app only, or multi-component interventions including an app as one of several intervention components. Outcomes measured were changes in the health behaviours and related health outcomes (i.e., fitness, body weight, blood pressure, glucose, cholesterol, quality of life). Study inclusion and methodological quality were independently assessed by two reviewers.
\end{abstract}

Results: Twenty-seven studies were included, most were randomised controlled trials ( $n=19 ; 70 \%)$. Twenty-three studies targeted adults (17 showed significant health improvements) and four studies targeted children (two demonstrated significant health improvements). Twenty-one studies targeted physical activity (14 showed significant health improvements), 13 studies targeted diet (seven showed significant health improvements) and five studies targeted sedentary behaviour (two showed significant health improvements). More studies $(n=12 ; 63 \%)$ of those reporting significant effects detected between-group improvements in the health behaviour or related health outcomes, whilst fewer studies $(n=8 ; 42 \%)$ reported significant within-group improvements. A larger proportion of multi-component interventions ( 8 out of $13 ; 62 \%$ ) showed significant between-group improvements compared to stand-alone app interventions (5 out of 14; 36\%). Eleven studies reported app usage statistics, and three of them demonstrated that higher app usage was associated with improved health outcomes.

Conclusions: This review provided modest evidence that app-based interventions to improve diet, physical activity and sedentary behaviours can be effective. Multi-component interventions appear to be more effective than standalone app interventions, however, this remains to be confirmed in controlled trials. Future research is needed on the optimal number and combination of app features, behaviour change techniques, and level of participant contact needed to maximise user engagement and intervention efficacy.

Keywords: Systematic review, Literature searches, Smartphone applications, Programs, Efficacy, Healthy eating, Exercise, Sitting, Health behaviour

\footnotetext{
* Correspondence: s.schoeppe@cqu.edu.au

${ }^{1}$ Central Queensland University, School of Health, Medical and Applied

Sciences, Physical Activity Research Group, Building 77, Bruce Highway,

Rockhampton QLD 4702, Australia

Full list of author information is available at the end of the article
}

(c) The Author(s). 2016 Open Access This article is distributed under the terms of the Creative Commons Attribution 4.0 International License (http://creativecommons.org/licenses/by/4.0/), which permits unrestricted use, distribution, and reproduction in any medium, provided you give appropriate credit to the original author(s) and the source, provide a link to the Creative Commons license, and indicate if changes were made. The Creative Commons Public Domain Dedication waiver (http://creativecommons.org/publicdomain/zero/1.0/) applies to the data made available in this article, unless otherwise stated. 


\section{Background}

The prevention of non-communicable diseases (NCDs) such as cardiovascular disease, cancer and type 2 diabetes is a major public health goal worldwide [1]. In 2012, NCDs were responsible for 38 million (68\%) of the world's 56 million deaths [1]. Lifestyle behaviours including diet, physical activity and sedentary behaviour are key modifiable risk factors for these diseases and improving these behaviours is considered essential to reducing the financial and health burden of these NCDs [1]. Unhealthy diet, physical inactivity and sedentary behaviour are known to track from childhood into adulthood and are difficult to change later in life $[2,3]$. This exacerbates associated health problems and demonstrates why preventing the development of these health risk factors throughout the lifespan is important.

Given the global scale of NCDs, preventative interventions that can reach large populations at low cost are needed. Smartphones and tablets, including the software applications (apps) that run on these devices, have become an integral part of people's life with large increases in usage rates since their introduction in 2007 [4, 5]. For example, $70 \%$ of Americans, $61 \%$ of Europeans and $74 \%$ of Australians regularly use a smartphone and/or tablet $[5,6]$. The growth in mobile technologies has also stimulated the growth in health and fitness apps to provide behavioural interventions that can reach large populations [7]. Clever use of apps in health promotion and prevention of disease has enabled researchers to apply health behaviour changes techniques (e.g., goal setting, self-monitoring, performance feedback) that have proven to facilitate health behaviour change across diverse population groups and settings $[7,8]$.

Despite the increasing use of apps in health behaviour change studies their efficacy is unclear, particularly for different lifestyle behaviours such as diet, physical activity and sedentary behaviour, as well as for specific population groups including children and adults. Some systematic reviews [9-13] have addressed the potential of health apps to improve diet, physical activity and/or sedentary behaviour. However, the scope of these reviews has been broad and many questions remain. Firstly, most previous reviews [9-11, 14] were on electronic and mobile health (e-\& mHealth) interventions, not specifically on app-based interventions. Secondly, many reviews $[7,8,15-18]$ have focused on app design, the incorporation of established behaviour change techniques and they examined intervention feasibility rather than efficacy. Thirdly, many previous reviews [19-22] have focused on the use of apps for the treatment of obesity and chronic diseases. As such, little is known about the potential of using apps to change health behaviours for disease prevention. Finally, no reviews have distinguished between app interventions for children versus adults. This is important as app features appealing to children and adolescents may differ from those used by adults, and the choice of app features will likely determine user engagement, retention and ultimately intervention efficacy [22].

This review aimed to address these gaps in the literature by systematically synthesising evidence for the efficacy of interventions that use apps to improve diet, physical activity and sedentary behaviour for NCD prevention. Since app features used by children may differ from those used by adults, we distinguished between app interventions targeted to children and adults.

\section{Methods}

\section{Literature search}

This review was conducted and reported according to the Preferred Reporting Items for Systematic Reviews and Meta-Analyses (PRISMA) guidelines (Additional file 1) [23]. Systematic searches were conducted between November and December 2015 in five databases: Scopus, CINAHL, SportDiscuss, PsycINFO and Web of Science. The search was limited to English language literature, humans, and the year of publication between 01 January 2006 and 31 October 2016. It was considered unlikely that app interventions were developed before 2006/2007 when smartphones were introduced. Systematic search strategies were designed using a combination of thesaurus and free terms covering the following terms: application, app, smartphone, smart phone, tablet, mobile game, game, physical activity, walk, physical fitness, leisure activity, motor activity, exercise, sport, sedentary, sedentary behaviour, sedentary behavior, sitting, screen time, inactive, diet, dietary, nutrition, nutritional, healthy eating, food, fruit, vegetable, snack, soft drink, carbonated beverage, intervention, program, programme, health promotion, prevention and trial. The detailed search strategies used for each database are presented in the Additional file 2. Additionally, articles were identified via hand-searching and reviewing reference lists of relevant papers.

\section{Inclusion criteria and study selection}

Studies were included in the present review if (1) they used an app in an intervention to influence at least one of the following lifestyle behaviours: dietary intake, physical activity, sedentary behaviour; (2) targeted children and/or adults; (3) focused on behaviour change for disease prevention (i.e., not specifically aiming to treat and manage health conditions such as obesity, hypertension and NCDs); and (4) reported data regarding efficacy for behaviour change (e.g., change in daily minutes of physical activity). In addition, studies could also report (but were not required) other relevant outcomes that may 
have conceivably been impacted by health behaviour change (i.e., fitness, body weight, blood pressure, glucose, cholesterol, quality of life). All types and units of measurements for the lifestyle behaviours and related health outcomes were acceptable (e.g., objective measure, self-report, minutes, steps, servings, calories, kilograms). The app intervention could be a stand-alone intervention using apps only, or a multi-component intervention where the use of an app was one of several intervention components (e.g., physical education, provision of physical activity equipment, parental education, face-to-face counselling). Studies were excluded from the review if: (1) non-experimental study designs were utilised (i.e., observational or case studies, studies reporting prevalence or trend data, feasibility studies, measurement studies, theoretical papers); and (2) the publication was not a peer-reviewed primary study (i.e., letters, commentaries, conference proceedings, reviews, narrative articles). Initially, titles and abstracts were screened for inclusion by a single reviewer (SS). As per best practice for systematic reviews [23], two reviewers (SA, WVL) independently reviewed the eligibility of studies for inclusion in the review, with disagreement resolved by discussion and consensus with a third reviewer (SS).

\section{Data extraction}

Data extraction was conducted using a standardised form developed specifically for this review (Table 1); similar to those used in other systematic reviews [12, 24, 25]. For all included studies, data were extracted for author, year, country, study design, intervention duration, measurement time points, attrition rate, sample, behaviour change theory, app features including behaviour change techniques, intervention components, outcomes, measures and main study results. The primary outcome measures extracted for main study results were dietary intake, physical activity and sedentary behaviour. Other relevant outcome measures closely related to these behaviours included weight status (body mass index, body weight, waist circumference), fitness, blood pressure, cholesterol, glucose and quality of life. To determine whether the interventions had a significant effect on behavioural and health outcomes, data on significance and magnitude of within- and between-group differences was extracted. For each included study, two reviewers independently extracted data (NAB and either SS, SA or WVL). Reviewers one and two agreed on the data extraction in over $70 \%$ of the studies. Disagreement was easily resolved by discussion and consensus with a third reviewer (either SS, SA or WVL).

\section{Study quality assessment}

The quality of the included studies was assessed using 25-point criteria adapted from the CONSORT checklists for the reporting of randomised controlled trials [26]. While the CONSORT checklist is intended for controlled trials, most criteria are applicable to other study designs and the weaker study designs justifiably received a lower score than studies using a controlled trial design. This approach has been used in other reviews [24]. Each criterion was rated as 1 (fulfilled), 0.5 (not all sub-items making up the criterion were fulfilled), 0 (not fulfilled or unclear), or not applicable (criterion was not applicable to the study design). Not applicable criteria were discounted from the 'overall study quality score' (sum of points). Hence, the highest attainable quality score was not 25 for all studies. Adapted from previous reviews $[27,28]$, the obtained study quality score for each study was divided by the highest attainable score and multiplied by 100 to give a percentage of fulfilled criteria; and studies were then grouped into high (>66.7\%), fair (50$66.6 \%)$ or low $(<50 \%)$ study quality (Additional file 3 ). The study quality assessment was conducted independently by two reviewers (SS, SA), with disagreement resolved by discussion and consensus with a third reviewer (WVL). Percent agreement between reviewers one and two for the scoring of the CONSORT criteria was $89 \%$, with the most common points of discrepancy relating to recruitment methods, outcomes reporting and blinding procedures.

\section{Results \\ Study selection}

A flowchart of the study selection process is presented in Fig. 1. A total of 6926 publications were identified from the database search. After removal of duplicates, 4945 publication titles and abstracts were screened, and 194 full-text articles were considered potentially eligible for inclusion. Of these, 30 articles reporting data on the efficacy of an app-based intervention to improve diet, physical activity and/or sedentary behaviour for prevention were included for final review. Some articles [29-34] reported on the same studies, and as such, a total of 30 articles describing 27 studies were included in this review.

\section{Study characteristics}

Characteristics of the app intervention studies included in this review are presented in Table 1.

Twelve studies were conducted in North America [35-46], seven studies in Australia/New Zealand [29-32, 47-51], seven studies in Europe [33, 34, 52-57], and one study in the Middle-East [58]. Most studies were randomised controlled trials $(n=19)$ with 2-group [29, 31, $35,40,43-46,48,50,52-55,58]$ or 3 -group [33, 34, $42,47,56,57]$ study designs. The remaining studies were controlled trials $(n=3)[33,37,49]$, randomised trials $(n=1)$ [39] or pre-post studies $(n=4)$ [36, 38, 41]. 
Table 1 Characteristics of the app-based intervention studies included in the review

\begin{tabular}{|c|c|c|}
\hline $\begin{array}{l}\text { Author } \\
\text { Year } \\
\text { Country }\end{array}$ & $\begin{array}{l}\text { Study design } \\
\text { Duration } \\
\text { Sample }\end{array}$ & $\begin{array}{l}\text { Behaviour change theory } \\
\text { App features } \\
\text { Intervention }\end{array}$ \\
\hline $\begin{array}{l}\text { Partridge et al. } 2015 \text { [31]; } \\
\text { Allman-Farinelli et al. } 2016 \\
\text { [32] } \\
\text { Australia }\end{array}$ & $\begin{array}{l}\text { Study design } \\
\text { 2-group RCT } \\
\text { Duration } \\
\text { Intervention exposure: } 12 \text { weeks } \\
\text { (Nov } 2012 \text { - July 2014) } \\
\text { Measurement points: baseline, } 12 \text { weeks, } \\
9 \text { months } \\
\text { Attrition rate: } 14 \% \\
\text { Sample } \\
\text { Adults } \\
N=250 \text { ( } 248 \text { analysed) } \\
27.7 \text { years/18-35 years } \\
39 \%(M), 61 \%(F) \\
\text { Random }\end{array}$ & $\begin{array}{l}\text { Behaviour change theory } \\
\text { Transtheoretical model } \\
\text { App features } \\
\text { Newly designed apps: } \\
4 \text { apps (one per behaviour); self- } \\
\text { monitoring of behaviours, educational } \\
\text { information, social networking through } \\
\text { community blog, informational support } \\
\text { resources } \\
\text { Intervention group } \\
\text { Used the apps; received eight text } \\
\text { messages and } 1 \text { email weekly, five } \\
\text { personalised coaching calls, a diet } \\
\text { booklet and access to resources and } \\
\text { four apps via a website } \\
\text { Control group } \\
\text { Received introductory call at week } 0, \\
\text { four text messages in total, and printed } \\
\text { dietary and physical activity guidelines } \\
\text { Multi-component versus stand-alone app } \\
\text { intervention } \\
\text { Multi-component }\end{array}$ \\
\hline
\end{tabular}

$\begin{array}{ll}\text { Outcomes } & \text { Results } \\ \text { Measures } & \text { Study quality }\end{array}$

Outcome

Physical activity (MET minutes/week, At 12 weeks, significant betweendays/week) group effects in vegetable

Diet (daily fruit and vegetable intake, $\quad(p=0.009)$, fast food $(p=0.01)$ and weekly fast food and sugar- sugar-sweetened beverages sweetened beverages intake) $\quad(p=0.002)$ intake in favour of the $\begin{array}{ll}\text { sweetened beverages intake) } & (p=0.002) \text { intake in favour of the } \\ \text { Other relevant outcomes } & \text { intervention group. Significant }\end{array}$ Weight status (body weight, BMI) between-group improvements were Measures

Physical activity (self-report:

International Physical Activity Questionnaire)

Diet (self-report, questionnaire)

Weight status (objectively measured height and weight)

sustained at 9 months follow-up

No significant between- group

difference in fruit intake.

Physical activity

At 12 weeks, significant between-

group increase in total physical

activity by 1.3 days/week ( $95 \% \mathrm{Cl}$ :

$0.5-2.2, P=0.003$ ) in intervention

group compared to control group.

No significant between- group

difference at 9 months follow-up.

Weight status

At 12 weeks, significant between-

group effects in body weight and

BMl: Participants in the intervention

group were $2.2 \mathrm{~kg}$ lighter compared

to the control group ( $95 \% \mathrm{Cl}: 0.8-3.6$

$p=0.005)$; and they had a $0.5 \mathrm{~kg} / \mathrm{m} 2$

lower BMI (95\% Cl: 0.1-1.0, $p=0.02$ ).

Significant between-group

improvements were sustained at

9 months follow-up.

Study quality

High

CONSORT score: 21

Percentage of fulfilled criteria: $85.7 \%$

Physical activity

$\begin{array}{ll}\text { Behaviour change theory } & \text { Outcome } \\ \text { Social cognitive theory } & \text { Physical activity (weekly steps }\end{array}$

Study design
2-group RCT

Duration
Duroup

App features

Intervention exposure: 12 weeks

$\begin{array}{ll}\text { Measurement points: baseline, } 12 \text { weeks } & \text { Fitbit app and tracker; includes self- } \\ \text { Attrition rate: } 17 \% & \text { monitoring of steps, performance }\end{array}$

Attrition rate: $17 \%$

Sample

counts)

None

Measures

Fitbit accelerometer

Intervention group

Questionnaires

No significant changes in physical activity.

Study quality

High

CONSORT score: 17.5

$\mathrm{N}=35 \quad$ Initial informational/educational session

33.7 years/18-40 years $\quad$ including specific goal setting and

gestational weight gain and safety

$100 \%$ (F)

instructions for promoting 
Table 1 Characteristics of the app-based intervention studies included in the review (Continued)

\begin{tabular}{|c|c|c|c|c|}
\hline & & $\begin{array}{l}\text { physical activity during pregnancy; used } \\
\text { Fitbit app and tracker; tips for physical } \\
\text { activity and healthy diet via the app; } \\
\text { daily messages (either text or short } \\
\text { videos) and activity diary, images and } \\
\text { short video clips regarding posture and } \\
\text { stretching, all via the app } \\
\text { Control group } \\
\text { Used only the Fitbit tracker, initial brief } \\
\text { in-person session, received IOM } \\
\text { recommendations } \\
\text { for gestational weight gain and safety } \\
\text { instruction for promoting } \\
\text { physical activity during pregnancy } \\
\text { Multi-component versus stand-alone app } \\
\text { intervention } \\
\text { Multi-component }\end{array}$ & & \\
\hline $\begin{array}{l}\text { Cowdery et al. } 2015 \text { [35] } \\
\text { USA }\end{array}$ & $\begin{array}{l}\text { Study design } \\
\text { 2-group RCT } \\
\text { Duration } \\
\text { Intervention exposure: } 12 \text { weeks } \\
\text { (Sept- Nov 2014) } \\
\text { Measurement points: baseline, } 12 \text { weeks } \\
\text { Attrition rate: } 4 \% \\
\text { Sample } \\
\text { Adults } \\
N=40 \\
\text { Median } 32.0 \text { years/18-69 years } \\
15 \%(M), 85 \%(F) \\
\text { Random }\end{array}$ & $\begin{array}{l}\text { Behaviour change theory } \\
\text { Self-determination theory } \\
\text { App features } \\
\text { Commercially available apps: } \\
\text { Gamification via immersive exergame } \\
\text { apps (Zombies Run!, The Walk) that } \\
\text { instruct users to run and exercise as part } \\
\text { of an audio adventure game, self- } \\
\text { monitoring and performance feedback } \\
\text { via physical activity tracking app } \\
\text { (MOVES) that monitors physical activity } \\
\text { frequency, duration, intensity and } \\
\text { distance } \\
\text { Intervention group } \\
\text { Use of one of the exergame apps, self- } \\
\text { monitoring of physical activity through } \\
\text { the MOVES app, weekly motivational } \\
\text { emails to increase intrinsic motivation } \\
\text { for physical activity based on self- } \\
\text { determination theory } \\
\text { Control group } \\
\text { Used only physical activity tracking app } \\
\text { (MOVES) } \\
\text { Multi-component versus stand-alone app } \\
\text { intervention } \\
\text { Multi-component }\end{array}$ & $\begin{array}{l}\text { Outcome } \\
\text { Physical activity (moderate, vigorous } \\
\text { and total physical activity, and } \\
\text { walking; minutes/week) } \\
\text { Other relevant outcomes } \\
\text { Weight status (BMI) } \\
\text { Blood pressure (diastolic and systolic) } \\
\text { Measures } \\
\text { Accelerometer (physical activity } \\
\text { tracking app MOVES) } \\
\text { Questionnaires } \\
\text { Blood pressure (monitor Omron-BP- } \\
760 \text { ) }\end{array}$ & $\begin{array}{l}\text { Physical activity } \\
\text { No significant changes in physical } \\
\text { activity. } \\
\text { Blood pressure } \\
\text { No significant changes in blood } \\
\text { pressure. } \\
\text { Weight status } \\
\text { No significant changes in BMI. } \\
\text { Study quality } \\
\text { Fair } \\
\text { CONSORT score: } 13.5 \\
\text { Percentage of fulfilled criteria: } 56.3 \%\end{array}$ \\
\hline $\begin{array}{l}\text { Direito et al. } 2015 \text { [47] } \\
\text { New Zealand }\end{array}$ & $\begin{array}{l}\text { Study design } \\
\text { 3-group RCT } \\
\text { Duration } \\
\text { Intervention exposure: } 8 \text { weeks } \\
\text { (June-Sept 2014) } \\
\text { Measurement points: baseline, } 8 \text { weeks } \\
\text { Attrition rate: } 8 \%\end{array}$ & $\begin{array}{l}\text { Behaviour change theory } \\
\text { Not reported } \\
\text { App features } \\
\text { Commercially available apps: } \\
\text { App 1: Immersive exergame app } \\
\text { (Zombies, Run!) that provides a training } \\
\text { program to improve fitness and ability }\end{array}$ & $\begin{array}{l}\text { Outcome } \\
\text { Physical activity (daily light, } \\
\text { moderate-to-vigorous, total) } \\
\text { Sedentary behaviour (total sedentary } \\
\text { time minutes/day) } \\
\text { Other relevant outcomes } \\
\text { Cardiorespiratory fitness }\end{array}$ & $\begin{array}{l}\text { Physical activity } \\
\text { No significant changes in light PA, } \\
\text { MVPA and overall PA. } \\
\text { Sedentary behaviour } \\
\text { No significant changes in sedentary } \\
\text { time. } \\
\text { Fitness }\end{array}$ \\
\hline
\end{tabular}


Table 1 Characteristics of the app-based intervention studies included in the review (Continued)

\begin{tabular}{|c|c|c|c|c|}
\hline & $\begin{array}{l}\text { Sample } \\
\text { Children } \\
N=51 \\
15.7 \text { years/14-17 years } \\
43 \%(\mathrm{M}), 57 \%(\mathrm{~F}) \\
\text { Random }\end{array}$ & $\begin{array}{l}\text { to run } 5 \text { km, information on running } \\
\text { and technique, audio instructions on } \\
\text { how to perform training components, } \\
\text { tracked and displayed progress, social } \\
\text { networking } \\
\text { App 2: non-immersive app (Get } \\
\text { Running) that provides an automated } \\
\text { training program to improve fitness and } \\
\text { ability to run } 5 \mathrm{~km} \text {, self-monitoring } \\
\text { (steps via pedometer), performance } \\
\text { feedback via app } \\
\text { Intervention group 1: } \\
\text { Used exergame app (Zombies, Run!) to } \\
\text { improve fitness and ability to run } 5 \text { km: } \\
\text { game-themed whereby the training } \\
\text { program was embedded with a story } \\
\text { where the user is trained to collect } \\
\text { supplies and protect a town from } \\
\text { zombies, self-monitoring, performance } \\
\text { feedback via app } \\
\text { Intervention group 2: } \\
\text { Used non-immersive app (Get Running) } \\
\text { that provides an automated training } \\
\text { program to improve fitness and ability } \\
\text { to run } 5 \text { km, self-monitoring, } \\
\text { performance feedback via app } \\
\text { Control group } \\
\text { Doing usual physical activities } \\
\text { Multi-component versus stand-alone app } \\
\text { intervention } \\
\text { Stand-alone }\end{array}$ & $\begin{array}{l}\text { Measures } \\
\text { Cardiorespiratory fitness ( } 1 \text { mile run/ } \\
\text { walk test) } \\
\text { Physical activity (questionnaires, } \\
\text { accelerometer worn on } 7 \\
\text { consecutive days during waking } \\
\text { hours) }\end{array}$ & $\begin{array}{l}\text { No significant changes in } \\
\text { cardiorespiratory fitness. } \\
\text { Study quality } \\
\text { High } \\
\text { CONSORT score: } 21 \\
\text { Percentage of fulfilled criteria: } 87.5 \%\end{array}$ \\
\hline $\begin{array}{l}\text { Elbert et al. } 2016 \text { [57] } \\
\text { Netherlands }\end{array}$ & $\begin{array}{l}\text { Study design } \\
\text { 3-group RCT } \\
\text { Duration } \\
\text { Intervention exposure: } 6 \text { months } \\
\text { Measurement points: baseline, } 6 \text { months } \\
\text { Attrition rate: } 57 \% \\
\text { Sample } \\
\text { Adults } \\
N=342 \text { (146 analysed) } \\
41.4 \text { years/16-71 } \\
27 \%(\mathrm{M}), 73 \% \text { (F) } \\
\text { Random }\end{array}$ & $\begin{array}{l}\text { Behaviour change theory } \\
\text { Not reported } \\
\text { App features } \\
\text { Newly designed app: } \\
\text { Fruit and Vegetables hAPP: Includes } \\
\text { tailored educational information via } \\
\text { either text or audio messages, action } \\
\text { planning, fruit and vegetable examples } \\
\text { and recipes } \\
\text { Intervention group } 1 \\
\text { Monthly text-based tailored health } \\
\text { information/messages delivered via the } \\
\text { app; used all other app features; } \\
\text { received unique testimonials } \\
\text { (constructed stories) via the app in } \\
\text { which successful personal experiences } \\
\text { were shared to encourage participants } \\
\text { to perform the behaviour themselves }\end{array}$ & $\begin{array}{l}\text { Outcome } \\
\text { Diet (fruit and vegetable intake/ } \\
\text { servings per week during previous } \\
\text { month) } \\
\text { Other relevant outcomes } \\
\text { None } \\
\text { Measures } \\
\text { Diet (Food-Frequency Questionnaire) }\end{array}$ & $\begin{array}{l}\text { Diet } \\
\text { Main effect analyses: Significant } \\
\text { between-group improvement in fruit } \\
\text { intake ( } F 2,140=3.08, p=0.049 \text { : text- } \\
\text { based app group (mean servings } \\
13.5, \text { SE 1.0), audio-based app group } \\
\text { (mean servings } 17.1, \text { SE } 1.2 \text { ), and } \\
\text { control group (mean servings } 14.3 \text {, } \\
\text { SE 0.9). However, the significant } \\
\text { difference occurred between IG } \\
1 \text { (text-based app) and IG2 (audio- } \\
\text { based app) }(p=0.02 \text { ), but no } \\
\text { significant differences between } \\
\text { control group and either of the } \\
\text { intervention groups. } \\
\text { No significant changes in vegetable } \\
\text { intake. }\end{array}$ \\
\hline
\end{tabular}

Outcome

month

None

Measures

and recipes

Monthly text-based tailored health

information/messages delivered via the

to perform the behaviour themselves 
Table 1 Characteristics of the app-based intervention studies included in the review (Continued)

\begin{tabular}{|c|c|c|c|}
\hline & & $\begin{array}{l}\text { Intervention group } 2 \\
\text { Monthly audio-based tailored health } \\
\text { information/messages from a female } \\
\text { actor delivered via the app; used all } \\
\text { other app features; received unique } \\
\text { testimonials (constructed stories) via the } \\
\text { app in which successful personal } \\
\text { experiences are shared to encourage } \\
\text { participants to perform the behaviour } \\
\text { themselves } \\
\text { Control group } \\
\text { No intervention } \\
\text { Multi-component versus stand-alone app } \\
\text { intervention } \\
\text { Stand-alone }\end{array}$ & \\
\hline $\begin{array}{l}\text { Fukuoka et al. } 2010 \text { [36] } \\
\text { USA }\end{array}$ & $\begin{array}{l}\text { Study design } \\
\text { Pre-post within-subjects design } \\
\text { Duration } \\
\text { Intervention exposure: } 3 \text { weeks } \\
\text { (June-Sept 2008) } \\
\text { Measurement points: Baseline, } 3 \text { weeks } \\
\text { Attrition rate: } 0 \% \\
\text { Sample } \\
\text { Adults } \\
N=41 \\
48.0 \text { years/25-40 years } \\
100 \% \text { (F) } \\
\text { Convenience }\end{array}$ & $\begin{array}{l}\text { Behaviour change theory } \\
\text { Not reported } \\
\text { App features } \\
\text { Newly designed app: } \\
\text { Goal setting, self-monitoring of physical } \\
\text { activity (step diary), motivational } \\
\text { messages } \\
\text { Intervention group } \\
\text { Used the app, used pedometer, received } \\
\text { daily prompts regarding benefits of } \\
\text { physical activity and social support } \\
\text { options } \\
\text { Control group } \\
\text { None } \\
\text { Multi-component versus stand-alone app } \\
\text { intervention } \\
\text { Multi-component }\end{array}$ & $\begin{array}{l}\text { Outcome } \\
\text { Physical activity (daily steps, daily } \\
\text { aerobic steps and } \mathrm{kcal} / \mathrm{kg} / \mathrm{day} \text { ) } \\
\text { Other relevant outcomes } \\
\text { None } \\
\text { Measures } \\
\text { Physical activity (pedometer and self- } \\
\text { reported questionnaire: } 7 \text {-day } \\
\text { Physical Activity Recall) }\end{array}$ \\
\hline $\begin{array}{l}\text { Garde et al. } \\
2015 \text { [37] } \\
\text { Canada }\end{array}$ & $\begin{array}{l}\text { Study design } \\
\text { 2-group CT } \\
\text { Duration } \\
\text { Intervention exposure: } 1 \text { week } \\
\text { Measurement points: baseline, during } \\
\text { intervention } \\
\text { Attrition rate: } 13 \% \\
\text { Sample } \\
\text { Children } \\
N=54 \text { ( } n=47 \text { analysed) } \\
10.0 \text { years/8-13 years } \\
16 \%(M), 84 \%(F) \\
\text { Convenience }\end{array}$ & $\begin{array}{l}\text { Behaviour change theory } \\
\text { Self-determination theory } \\
\text { App features } \\
\text { Newly designed app: } \\
\text { App (Mobilekids Monster Manor), } \\
\text { physical activity earns gaming currency } \\
\text { (gamification), inter-team competition, } \\
\text { peer support } \\
\text { Intervention group } \\
\text { Used the app } \\
\text { Control group } \\
\text { Received daily physical activity feedback } \\
\text { via a website } \\
\text { Multi-component versus stand-alone app } \\
\text { intervention } \\
\text { Stand-alone }\end{array}$ & $\begin{array}{l}\text { Outcome } \\
\text { PA (steps/day, minutes/day) } \\
\text { Other relevant outcomes } \\
\text { None } \\
\text { Measures } \\
\text { Physical activity (accelerometer- } \\
\text { based activity monitor Tractivity) }\end{array}$ \\
\hline
\end{tabular}

Moderation effect analyses: In adults with poor perceived own health status, significant-between-group differences in fruit intake in favour of IG2 (F2,137 =6.05, $p=0.003$, partial $\eta 2=0.08)$; mean fruit servings were (IG1: 14.2), (IG2: 20.5) and (CG: 13.2) Fruit intake was significantly higher in IG2 compared to IG1 $(p=0.006)$ and CG $(p=0.001)$.

Study quality

CONSORT score: 14.5

Percentage of fulfilled criteria: $60.4 \%$

Physical activity

Significant increase in mean daily steps from baseline $(5394 ; 95 \% \mathrm{Cl}$ : 4563-6224) to 3 weeks (6210; 95\% Cl: 5379-7041) $(p=0.001)$

Significant increase in mean aerobic steps from baseline $(953.95 \% \mathrm{Cl}$

489-1416) to 3 weeks $(1535 ; 95 \% \mathrm{Cl}$ : 1074-1996) $(p<0.001)$

Significant increase in $\mathrm{kcal} / \mathrm{kg} / \mathrm{day}$

from baseline $(32.5 \pm 1.28)$ to 3 weeks $(33.4 \pm 1.99)(p=0.01)$. Study quality

Low

CONSORT score: 11.5

Percentage of fulfilled criteria: $46.9 \%$

Physical activity

No significant between-group

changes in physical activity.

Significant within-group increase in

IG in relation to steps/day (1191;

$p=0.01)$ and active minutes/day

$(25 ; p=0.03)$

Study quality

CONSORT score: 16

Percentage of fulfilled criteria: $65.3 \%$ 
Table 1 Characteristics of the app-based intervention studies included in the review (Continued)

\begin{tabular}{|c|c|c|c|c|}
\hline $\begin{array}{l}\text { Gasser et al. } 2015 \\
\text { Switzerland }\end{array}$ & $\begin{array}{l}\text { Study design } \\
\text { 2-group RCT } \\
\text { Duration } \\
\text { Intervention exposure: } 4 \text { weeks } \\
\text { Measurement points: baseline, } 4 \text { weeks } \\
\text { Attrition rate: not reported } \\
\text { Sample } \\
\text { Children and adults } \\
N=40 \text { ( } 39 \text { analysed) } \\
32.0 \text { years/14-50 years } \\
50 \% \text { (M), 50\% (F) } \\
\text { Random (but age and gender } \\
\text { controlled) }\end{array}$ & $\begin{array}{l}\text { Behaviour change theory } \\
\text { Not reported } \\
\text { App features } \\
\text { Newly designed app: } \\
\text { Self-monitoring of physical activity and } \\
\text { food consumption (via diary in app), } \\
\text { goal-setting, individual and team } \\
\text { performance feedback on daily goal } \\
\text { achievement, social support (teams), } \\
\text { received messages, reminders and } \\
\text { questionnaires } \\
\text { Intervention group } \\
\text { Used smartphone app } \\
\text { Control group } \\
\text { Used a similar web-based app that } \\
\text { worked on any operating system/ } \\
\text { browsers } \\
\text { Multi-component versus stand-alone app } \\
\text { intervention } \\
\text { Stand-alone }\end{array}$ & $\begin{array}{l}\text { Outcome } \\
\text { Physical activity (daily moderate-to- } \\
\text { vigorous) } \\
\text { Diet (fruit and vegetable } \\
\text { consumption, servings/day) } \\
\text { Other relevant outcomes } \\
\text { Weight status (BMI) } \\
\text { Measures } \\
\text { Online questionnaire }\end{array}$ & $\begin{array}{l}\text { Diet } \\
\text { No significant changes in fruit and } \\
\text { vegetable consumption. } \\
\text { Physical activity } \\
\text { No significant changes in physical } \\
\text { activity. } \\
\text { Weight status } \\
\text { No significant changes in BMI. } \\
\text { Study quality } \\
\text { Low } \\
\text { CONSORT score: } 8.5 \\
\text { Percentage of fulfilled criteria: } 34.0 \%\end{array}$ \\
\hline $\begin{array}{l}\text { Gilliland et al. } 2015 \text { [38] } \\
\text { Canada }\end{array}$ & $\begin{array}{l}\text { Study design } \\
\text { Pre-post within-subjects design } \\
\text { Duration } \\
\text { Intervention exposure: baseline, 8-10 } \\
\text { weeks } \\
\text { Measurement points: Baseline, post } \\
\text { intervention (varied weeks post baseline) } \\
\text { Attrition rate: } 44 \% \\
\text { Sample } \\
\text { Adults } \\
N=208 \\
33.0 \text { years/age range not reported } \\
34 \%(M), 66 \%(F) \\
\text { Convenience }\end{array}$ & $\begin{array}{l}\text { Behaviour change theory } \\
\text { Not reported } \\
\text { App features } \\
\text { Newly designed app: } \\
\text { App (SmartAPPetite) that includes } \\
\text { education on diet and health, goal } \\
\text { setting, rewards, motivational } \\
\text { interviewing, time management tips, } \\
\text { healthy eating tips, recipes, vendor } \\
\text { spotlights and coupons, behaviour- } \\
\text { health link } \\
\text { Intervention components } \\
\text { Used the app } \\
\text { Control group } \\
\text { None } \\
\text { Multi-component versus stand-alone app } \\
\text { intervention } \\
\text { Stand-alone }\end{array}$ & $\begin{array}{l}\text { Outcome } \\
\text { Diet (weekly consumption of health } \\
\text { food items) } \\
\text { Other relevant outcomes } \\
\text { None } \\
\text { Measures } \\
\text { Diet (self-report questionnaire) }\end{array}$ & $\begin{array}{l}\text { Diet } \\
\text { Significant correlation between app } \\
\text { use (check-ins) and food } \\
\text { consumption (vegetables } r=0.23 \text {; } \\
\text { soft drinks } r=-0.30 \text {, fruit juice } \\
r=-0.35 ; p<0.05 \text { ). } \\
\text { Study quality } \\
\text { Low } \\
\text { CONSORT score: } 8.5 \\
\text { Percentage of fulfilled criteria: } 34.7 \%\end{array}$ \\
\hline $\begin{array}{l}\text { Gilson et al. } 2016 \text { [51] } \\
\text { Australia }\end{array}$ & $\begin{array}{l}\text { Study design } \\
\text { Pre-post within-subjects design } \\
\text { Duration } \\
\text { Intervention exposure: } 20 \text { weeks } \\
\text { Measurement points: Baseline, weeks } 4 \text {, } \\
8,12,16,20 \\
\text { Attrition rate: } 57 \% \\
\text { Sample } \\
\text { Adults } \\
N=44 \text { (26 analysed) } \\
47.0 \text { years/age range not reported }\end{array}$ & $\begin{array}{l}\text { Behaviour change theory } \\
\text { Not reported } \\
\text { App features } \\
\text { Commercially available app: } \\
\text { Jawbone Up that includes self- } \\
\text { monitoring of daily step counts and } \\
\text { logging dietary choices; includes news } \\
\text { feeds, notifications and status updates, } \\
\text { can connect with other users } \\
\text { Intervention components } \\
\text { Used the app and received intervention }\end{array}$ & $\begin{array}{l}\text { Outcome } \\
\text { Diet (healthy dietary choices) } \\
\text { Physical activity (daily/weekly step } \\
\text { counts) } \\
\text { Other relevant outcomes } \\
\text { None } \\
\text { Measures } \\
\text { Diet (self-report questionnaire) }\end{array}$ & $\begin{array}{l}\text { Diet } \\
\text { No significant changes in healthy } \\
\text { diet choices. } \\
\text { Physical activity } \\
\text { No significant changes in step } \\
\text { counts. } \\
\text { Study quality } \\
\text { Low } \\
\text { CONSORT score: } 9.5 \\
\text { Percentage of fulfilled criteria: } 39.6 \%\end{array}$ \\
\hline
\end{tabular}


Table 1 Characteristics of the app-based intervention studies included in the review (Continued)

\begin{tabular}{|c|c|c|c|c|}
\hline & Convenience & $\begin{array}{l}\text { researchers through connecting via the } \\
\text { app } \\
\text { Control group } \\
\text { None } \\
\text { Multi-component versus stand-alone app } \\
\text { intervention } \\
\text { Stand-alone }\end{array}$ & & \\
\hline $\begin{array}{l}\text { Glynn et al. } 2014 \text { [53] } \\
\text { Ireland }\end{array}$ & $\begin{array}{l}\text { Study design } \\
\text { 2-group RCT } \\
\text { Duration } \\
\text { Intervention exposure: } 8 \text { weeks } \\
\text { (Aug } 2012 \text { - June 2103) } \\
\text { Measurement points: baseline, } 8 \text { weeks } \\
\text { Attrition rate: } 14 \% \\
\text { Sample } \\
\text { Children and adults } \\
N=90 \text { (77 analysed) } \\
44.1 \text { years/>16 years } \\
36 \%(M), 64 \% \text { (F) } \\
\text { Random }\end{array}$ & $\begin{array}{l}\text { Behaviour change theory } \\
\text { Not reported } \\
\text { App features } \\
\text { Commercially available app: } \\
\text { Used the Accupedo-Pro Pedometer app. } \\
\text { Goal setting functionality and goal } \\
\text { setting achievement feedback, self- } \\
\text { monitoring of step counts and calories } \\
\text { burnt, automatic performance feedback } \\
\text { through graphic display of step-count } \\
\text { history } \\
\text { Intervention group } \\
\text { Received physical activity goals (10,000 } \\
\text { steps/day) and information on the } \\
\text { benefits of exercise, smartphone app } \\
\text { and instruction on how to use it, } \\
\text { telephone mentoring sessions with } \\
\text { physical activity goal setting } \\
\text { Control group } \\
\text { Received physical activity goals (walking } \\
\text { for } 30 \text { min/day in addition to normal } \\
\text { activity) and information on benefits of } \\
\text { exercise but app not made visible on } \\
\text { their smartphone and no instructions on } \\
\text { how to use the app to achieve these } \\
\text { goals } \\
\text { Multi-component versus stand-alone app } \\
\text { intervention } \\
\text { Multi-component }\end{array}$ & $\begin{array}{l}\text { Outcome } \\
\text { Physical activity (steps/day) } \\
\text { Other relevant outcomes } \\
\text { Weight status (body weight, BMI) } \\
\text { Quality of life } \\
\text { Blood pressure (diastolic and systolic) } \\
\text { Measures } \\
\text { Physical activity (pedometer) } \\
\text { Weight status (objectively measured } \\
\text { height and weight) } \\
\text { Blood pressure (monitor) } \\
\text { Quality of life (questionnaires) }\end{array}$ & $\begin{array}{l}\text { Physical activity } \\
\text { Significant between-group increase } \\
\text { in mean steps/day in IG at } 8 \text { week } \\
\text { follow-up (1631 } \pm 3842 ; p=0.03 \text { ). } \\
\text { Weight status } \\
\text { No significant changes in body } \\
\text { weight. } \\
\text { No significant changes in BMI. } \\
\text { Blood pressure } \\
\text { No significant changes in blood } \\
\text { pressure. } \\
\text { Quality of life } \\
\text { No significant changes in quality of } \\
\text { life. } \\
\text { Study quality } \\
\text { High } \\
\text { CONSORT score: } 18 \\
\text { Percentage of fulfilled criteria: } 73.5 \%\end{array}$ \\
\hline $\begin{array}{l}\text { Hebden et al. } 2014 \text { [48] } \\
\text { Australia }\end{array}$ & $\begin{array}{l}\text { Study design } \\
\text { 2-group RCT } \\
\text { Duration } \\
\text { Intervention exposure: } 12 \text { weeks } \\
\text { (July- Dec 2011) } \\
\text { Measurement points: baseline, } \\
\text { weeks } 13 \\
\text { Attrition rate: } 10 \% \\
\text { Sample } \\
\text { Adults } \\
N=51 \\
23.0 \text { years/18-35 years } \\
20 \%(M), 80 \%(F) \\
\text { Random }\end{array}$ & $\begin{array}{l}\text { Behaviour change theory } \\
\text { Transtheoretical model } \\
\text { App features } \\
\text { Newly designed apps: } \\
4 \text { apps (one per behaviour); physical } \\
\text { activity self-monitoring, servings of fruit } \\
\text { and vegetables, energy and fat content } \\
\text { of take away meals and tailored advice } \\
\text { Intervention group } \\
\text { Used the apps, received SMS text and } \\
\text { email messages and internet forums } \\
\text { Control group } \\
\text { Printed diet booklet with instructions } \\
\text { from dietician. }\end{array}$ & $\begin{array}{l}\text { Outcome } \\
\text { Physical activity (light, MET; minutes/ } \\
\text { week) } \\
\text { Sedentary behaviour (sedentary time; } \\
\text { minutes/week) } \\
\text { Diet (daily fruit and vegetable intake } \\
\text { and weekly fast food consumption) } \\
\text { Other relevant outcomes } \\
\text { Weight status (body weight, } \\
\text { BMI) } \\
\text { Measures } \\
\text { Physical activity, sedentary behaviour } \\
\text { (self-report: International Physical }\end{array}$ & $\begin{array}{l}\text { Diet } \\
\text { No between- group change in fruit } \\
\text { and vegetable intake or } \\
\text { consumption of takeaway meals. } \\
\text { Physical activity } \\
\text { Significant between-group increase } \\
\text { in light intensity activity in IG at } \\
13 \text { week follow-up ( } 34.2 \pm 35.1, \\
p=0.001) \text {. No between group } \\
\text { differences for self-reported MET } \\
\text { minutes of physical activity. } \\
\text { Sedentary behaviour } \\
\text { No significant changes in sedentary } \\
\text { behaviour. }\end{array}$ \\
\hline
\end{tabular}


Table 1 Characteristics of the app-based intervention studies included in the review (Continued)

\begin{tabular}{|c|c|c|c|}
\hline & & $\begin{array}{l}\text { Multi-component versus stand-alone app } \\
\text { intervention } \\
\text { Multi-component }\end{array}$ & $\begin{array}{l}\text { Activity Questionnaire; } \\
\text { accelerometer) } \\
\text { Sitting time (self-report, } \\
\text { questionnaire) } \\
\text { Diet (self-report, takeaway and fruit } \\
\text { and vegetable consumption) } \\
\text { Weight status (objectively measured } \\
\text { height and weight) }\end{array}$ \\
\hline $\begin{array}{l}\text { King et al. } \\
2013 \text { [39] } \\
\text { USA }\end{array}$ & $\begin{array}{l}\text { Study design } \\
\text { 3-group randomised trial } \\
\text { Duration } \\
\text { Intervention exposure: } 8 \text { weeks } \\
\text { Measurement points: baseline, } 8 \text { weeks } \\
\text { Attrition rate: } 11 \% \\
\text { Sample } \\
\text { Adults } \\
N=68 \text { (N } 61 \text { analysed) } \\
59.1 \text { years/>45 years } \\
26 \% \text { (M), } 74 \% \text { (F) } \\
\text { Random }\end{array}$ & $\begin{array}{l}\text { Behaviour change theory } \\
\text { Social cognitive theory, social influence } \\
\text { theory } \\
\text { App features } \\
\text { Newly designed apps: } \\
3 \text { different apps: } \\
\text { 'Analytic' motivational app including } \\
\text { goal-setting and feedback, barriers } \\
\text { 'Social' motivational app including social } \\
\text { norms, modelling, competition and } \\
\text { collaboration. } \\
\text { 'Affective' motivational app including } \\
\text { positive reinforcement, modelling, } \\
\text { feedback and gamification. } \\
\text { In addition, all apps incorporated push } \\
\text { and pull components, glance-able } \\
\text { display, passive activity assessment, real } \\
\text { time feedback, self-monitoring, } \\
\text { reinforcement. } \\
\text { Intervention group } \\
\text { Used the apps } \\
\text { Control group } \\
\text { None } \\
\text { Multi-component versus stand-alone app } \\
\text { intervention } \\
\text { Stand-alone }\end{array}$ & $\begin{array}{l}\text { Outcome } \\
\text { Physical activity (brisk walking, } \\
\text { moderate-to-vigorous; minutes/ } \\
\text { week) } \\
\text { Sedentary behaviour (television } \\
\text { viewing; minutes/day) } \\
\text { Other relevant outcomes } \\
\text { None } \\
\text { Measures } \\
\text { Physical activity (self-report: CHAMPS } \\
\text { Physical Activity Questionnaire) } \\
\text { Sedentary behaviour (self -report: } \\
\text { Measure of Older Adults Sedentary } \\
\text { Time MOST) }\end{array}$ \\
\hline $\begin{array}{l}\text { Kirwan et al. } 2012 \text { [49] } \\
\text { Australia }\end{array}$ & $\begin{array}{l}\text { Study design } \\
\text { 2-group CT (matched case-control trial) } \\
\text { Duration } \\
\text { Intervention exposure: } 12 \text { weeks } \\
\text { (August-October 2009) } \\
\text { Measurement points: baseline, } 12 \text { weeks } \\
\text { Attrition rate: } 0 \% \\
\text { Sample } \\
\text { Adults } \\
N=200 \\
39.7 \text { years/17-64 years } \\
52 \%(M), 48 \% \text { (F) } \\
\text { Convenience }\end{array}$ & $\begin{array}{l}\text { Behaviour change theory } \\
\text { Not reported } \\
\text { App features } \\
\text { Newly designed app: } \\
\text { Self-monitoring of physical activity (steps } \\
\text { via iSteplog) } \\
\text { Intervention group } \\
\text { Participants logged steps using either } \\
\text { app or 10,000 steps website, goal- } \\
\text { setting, performance feedback } \\
\text { Control group } \\
\text { Participants logged steps using 10,000 } \\
\text { step website, but no access to iSteplog } \\
\text { app }\end{array}$ & $\begin{array}{l}\text { Outcome } \\
\text { Physical activity (steps/day) } \\
\text { Other relevant outcomes } \\
\text { None } \\
\text { Measures } \\
\text { Accelerometer }\end{array}$ \\
\hline
\end{tabular}

Weight status

No significant changes in weight status.

Study quality

High

CONSORT score: 19.5

Percentage of fulfilled criteria: $79.6 \%$

Physical activity

Significant within-group increases in mean minutes/week of brisk walking across all 3 app groups at 8 week follow-up (100.8 $\pm 167.0 ; p<0.001)$ Significant within-group increase in mean minutes/week of total MVPA across all 3 app groups at 8 week follow-up $(188.6 \pm 289.3 ; p<0.001)$ No significant between-group changes in physical activity. Sedentary behaviour

Significant within-group decrease in minutes/day spent sitting whilst watching television $(29.1 \pm 84.5$; $p<0.02$ ) across all 3 app groups at 8 week follow-up.

No significant between-group changes in sedentary behaviour. Study quality

Low

CONSORT score: 12

Percentage of fulfilled criteria: $46.9 \%$

Physical activity

Between group increase in steps/day in IG at 12 week follow-up $(11,140 . \pm$ 4,121vs CG: 6,274 $\pm 2,106, p<0.001)$. Study quality

CONSORT score: 14.5

Percentage of fulfilled criteria: $59.2 \%$ 
Table 1 Characteristics of the app-based intervention studies included in the review (Continued)

\begin{tabular}{|c|c|c|c|}
\hline & & $\begin{array}{l}\text { Multi-component versus stand-alone app } \\
\text { intervention } \\
\text { Multi-component }\end{array}$ & \\
\hline $\begin{array}{l}\text { Maher et al. } 2015 \text { [50] } \\
\text { Australia }\end{array}$ & $\begin{array}{l}\text { Study design } \\
\text { 2-group RCT } \\
\text { Duration } \\
\text { Intervention exposure: } 8 \text { weeks } \\
\text { (September } 2013 \text { - July 2014) } \\
\text { Measurement points: baseline, } 8 \text { weeks, } \\
20 \text { weeks } \\
\text { Attrition rate: 13\% } \\
\text { Sample } \\
\text { Adults } \\
N=110 \\
35.6 \text { years/18-65 years } \\
29 \% \text { (M), 71\% (F) } \\
\text { Random }\end{array}$ & $\begin{array}{l}\text { Behaviour change theory } \\
\text { Theory of planned behaviour, fun theory } \\
\text { App features } \\
\text { Newly designed app: } \\
\text { Facebook app (Active Team) including } \\
\text { goal setting ( } 10,000 \text { steps/day), self- } \\
\text { monitoring of physical activity (calendar } \\
\text { to log daily steps), performance } \\
\text { feedback via tally board to monitor } \\
\text { individual and teammates' progress; } \\
\text { team message board to allow team } \\
\text { members to communicate with one } \\
\text { another; gamification in the form of } \\
\text { awards for individual and team step- } \\
\text { logging and step-count achievement, as } \\
\text { well as sending virtual gifts to } \\
\text { teammates; peer social support through } \\
\text { Facebook friends (Active Teams) } \\
\text { Intervention group } \\
\text { Used the app, automated computer- } \\
\text { tailored emails to summarise progress } \\
\text { and encourage continued participation, } \\
\text { use of pedometer to encourage achieve } \\
\text { 10,000 steps/day } \\
\text { Control group } \\
\text { Wait-list control } \\
\text { Multi-component versus stand-alone app } \\
\text { intervention } \\
\text { Multi-component }\end{array}$ & $\begin{array}{l}\text { Outcome } \\
\text { Physical activity (moderate, vigorous, } \\
\text { walking; minutes/week) } \\
\text { Other relevant outcomes } \\
\text { Quality of life } \\
\text { Measures } \\
\text { Questionnaires }\end{array}$ \\
\hline $\begin{array}{l}\text { Mummah et al. } 2016 \text { [45] } \\
\text { USA }\end{array}$ & $\begin{array}{l}\text { Study design } \\
\text { 2-group RCT } \\
\text { Duration } \\
\text { Intervention exposure: } 12 \text { weeks } \\
\text { Measurement points: baseline, } 12 \text { weeks } \\
\text { Attrition rate: } 24 \% \\
\text { Sample } \\
\text { Adults } \\
N=17 \\
42.05 \text { years/18-50 years } \\
35 \%(\mathrm{M}), 65 \% \text { (F) } \\
\text { Random }\end{array}$ & $\begin{array}{l}\text { Behaviour change theory } \\
\text { Behavioural theory } \\
\text { App features } \\
\text { Newly designed app: } \\
\text { Goal setting for and self-monitoring of } \\
\text { vegetable consumption (i.e., vegetable } \\
\text { logging by tapping on different } \\
\text { vegetable icons and recording the } \\
\text { number of servings consumed); } \\
\text { performance feedback via graphs, social } \\
\text { comparison with friends via leaderboard, } \\
\text { consumption challenges delivered via } \\
\text { push notifications, prompts to log } \\
\text { vegetables via push notifications } \\
\text { Intervention group } \\
\text { Used the app } \\
\text { Control group } \\
\text { Wait-list control }\end{array}$ & $\begin{array}{l}\text { Outcome } \\
\text { Diet (daily vegetable consumption/ } \\
\text { servings) } \\
\text { Measures } \\
\text { Questionnaires (Food Frequency } \\
\text { Questionnaire) }\end{array}$ \\
\hline
\end{tabular}

Physical activity

8-week follow-up:

Significant between-group increase

in mean weekly minutes of overall

PA in $1 G(528 \pm 391$ vs CG: $391 \pm 371$

effect size: $0.39,95 \%$ Cl:0.01-0.76)

and walking (332 289 vs CG: $160 \pm$

185, effect size: $0.69,95 \%$ Cl: $0.30-$

$1.07)$

20-week follow-up:

Physical activity remained higher

compared to baseline, and higher in

IG compared to CG. But within-

group and between-group

differences were not significant.

Quality of life

No significant changes in quality of

life at 8-week and 20-week follow-

ups.

Study quality

High

CONSORT score: 19

Percentage of fulfilled criteria: $77.6 \%$

Diet

Significant between-group increase

in vegetable consumption in

intervention group compared to

control group (adjusted mean

control group (adjusted mean
difference: 7.4 servings; $95 \%$ Cl: $1.4-$

$13.5 ; p=0.02$ )

Study quality

High

CONSORT score: 17.5

Percentage of fulfilled criteria: $72.9 \%$ 
Table 1 Characteristics of the app-based intervention studies included in the review (Continued)

\begin{tabular}{|c|c|c|}
\hline & & $\begin{array}{l}\text { Multi-component versus stand-alone app } \\
\text { intervention } \\
\text { Stand-alone }\end{array}$ \\
\hline $\begin{array}{l}\text { Nollen et al. } 2014 \text { [40] } \\
\text { USA }\end{array}$ & $\begin{array}{l}\text { Study design } \\
\text { 2-group RCT } \\
\text { Duration } \\
\text { Intervention exposure: } 12 \text { weeks (weeks } \\
\text { 1-4: fruits/vegetables; weeks 5-8: sugar- } \\
\text { sweetened beverages; weeks 9-12: } \\
\text { screen time) } \\
\text { March 2011- April 2012 } \\
\text { Measurement points: baseline, } 4 \text { weeks } \\
\text { (fruits/vegetables), } 8 \text { weeks (sugar- } \\
\text { sweetened beverages), } 12 \text { weeks (screen } \\
\text { time) } \\
\text { Attrition rate: 14\% } \\
\text { Sample } \\
\text { Children } \\
\mathrm{N}=51 \\
11.3 \text { years/9-14 years } \\
100 \% \text { (F) } \\
\text { Random }\end{array}$ & $\begin{array}{l}\text { Behaviour change theory } \\
\text { Not reported } \\
\text { App features } \\
\text { Commercially available app: } \\
\text { Real-time goal setting, action planning, } \\
\text { self-monitoring and tips, feedback and } \\
\text { positive reinforcements on goal- } \\
\text { attainment through song-based rewards } \\
\text { system (received } 1 \text { song/day if girls } \\
\text { responded to } 80 \% \text { of daily prompts) } \\
\text { Intervention group } \\
\text { Used the app } \\
\text { Control group } \\
\text { Used the app but without action cues } \\
\text { and reward system } \\
\text { Multi-component versus stand-alone app } \\
\text { intervention } \\
\text { Stand-alone }\end{array}$ \\
\hline $\begin{array}{l}\text { Rabbi et al. } 2015 \text { [46] } \\
\text { USA }\end{array}$ & $\begin{array}{l}\text { Study design } \\
\text { 2-group RCT } \\
\text { Duration } \\
\text { Intervention exposure: } 3 \text { weeks } \\
\text { (randomisation after week 1) } \\
\text { Measurement points: baseline, week } 3 \\
\text { Attrition rate: } 6 \% \\
\text { Sample } \\
\text { Adults } \\
N=18 \text { (17 analysed) } \\
28.3 \text { years/18-49 years } \\
53 \%(\mathrm{M}), 47 \%(\mathrm{~F}) \\
\text { Random }\end{array}$ & $\begin{array}{l}\text { Behaviour change theory } \\
\text { Learning theory, social cognitive theory, } \\
\text { fogg behaviour model } \\
\text { App features } \\
\text { Newly designed app: } \\
\text { MyBehaviour app included self- } \\
\text { monitoring of physical activity, and food } \\
\text { and caloric intake; logging clusters/ } \\
\text { patterns of physical activities and foods; } \\
\text { prompting goal setting via automatic } \\
\text { generation of suggestions for exercise } \\
\text { and food based on logged activities and } \\
\text { food items. } \\
\text { Intervention group } \\
\text { Used the app, received 'tailored' } \\
\text { suggestions for exercise and food intake } \\
\text { via the app based on logged activities } \\
\text { and food items; face-to-face training } \\
\text { session on how to use the app } \\
\text { Control group } \\
\text { Used the app; received 'generic' } \\
\text { prescriptive recommendations for } \\
\text { physical activities and dietary intake } \\
\text { created by health professionals and } \\
\text { delivered via the app; face-to-face } \\
\text { training session on how to use the app }\end{array}$ \\
\hline
\end{tabular}

Outcome

Diet (fruit and vegetable

consumption, sugar-sweetened

beverages consumption)

Sedentary behaviour (screen time)

Other relevant outcomes

Weight status (BMI)

Measures

Diet (questionnaires: 24-h dietary

recall)

Sedentary behaviour (questionnaires: Brief Questionnaire of Television and Computer use)

BMI (objectively measured height and weight)

Outcome

Diet (caloric intake)

Physical activity (walking minutes/

week)

Dther relevant outcomes

None

Measures

Physical activity and diet (daily diary)
Diet

Between-group increase in fruit and

vegetable consumption in IG at

12 week follow-up, but not

significant ( $p=0.08$ )

Between-group decrease in sugar-

sweetened beverage consumption in

G at 12 week follow-up, but not

significant $(p=0.09)$

Sedentary behaviour

No significant changes in sedentary behaviour.

Weight status

No significant changes in weigh

status.

Study quality

CONSORT score: 12.5

Percentage of fulfilled criteria: $51.0 \%$

Diet

No significant changes in diet.

Physical activity

No significant changes in physical activity.

Study quality

CONSORT score: 14

Percentage of fulfilled criteria: $58.3 \%$ 
Table 1 Characteristics of the app-based intervention studies included in the review (Continued)

\begin{tabular}{|c|c|c|c|c|}
\hline & & $\begin{array}{l}\text { Multi-component versus stand-alone app } \\
\text { intervention } \\
\text { Stand-alone }\end{array}$ & & \\
\hline $\begin{array}{l}\text { Rospo et al. } 2016 \text { [56] } \\
\text { Italy }\end{array}$ & $\begin{array}{l}\text { Study design } \\
\text { 3-group randomised controlled trial } \\
\text { (only IG1 and IG2 were randomised) } \\
\text { Duration } \\
\text { Intervention exposure: } 2 \text { weeks } \\
\text { Measurement points: baseline, week 1, } \\
\text { week } 2 \\
\text { Attrition rate: } 27 \% \\
\text { Sample } \\
\text { Adults } \\
N=45 \text { ( } 33 \text { analysed) } \\
56.6 \text { years/20-55 years } \\
39 \%(M), 61 \%(F) \\
\text { Random }\end{array}$ & $\begin{array}{l}\text { Behaviour change theory } \\
\text { Not reported } \\
\text { App features } \\
\text { Newly designed app vs commercially } \\
\text { available app: } \\
\text { Both apps included self-monitoring, } \\
\text { performance feedback, goal setting. The } \\
\text { newly designed cardio fitness app } \\
\text { focused on heart rate monitoring in } \\
\text { particular } \\
\text { Intervention groups } \\
\text { IG1: Step-count app group } \\
\text { Used the Fitbit app, instructed to } \\
\text { complete } 10,000 \text { steps a day } \\
\text { IG2: Cardio fitness app group } \\
\text { Used the newly designed cardio fitness } \\
\text { app to receive performance feedback, } \\
\text { completed an fitness intensity training } \\
\text { based on the guidelines of the American } \\
\text { College of Sports Medicine } \\
\text { IG3: Supervised cardio fitness group } \\
\text { Completed an fitness intensity training } \\
3-4 \text { times/week at the gym based on } \\
\text { the guidelines of the American College } \\
\text { of Sports, received face-to-face } \\
\text { performance feedback } \\
\text { Control group } \\
\text { None } \\
\text { Multi-component versus stand-alone app } \\
\text { intervention } \\
\text { Stand-alone }\end{array}$ & $\begin{array}{l}\text { Outcome measures } \\
\text { Physical activity (steps/week) } \\
\text { Other relevant outcomes } \\
\text { Cardiorespiratory fitness (maximal } \\
\text { oxygen uptake) } \\
\text { Weight status (BMl } \mathrm{kg} / \mathrm{m}^{2} \text { ) } \\
\text { Blood pressure (diastolic and systolic; } \\
\mathrm{mm} \mathrm{Hg} \text { ) } \\
\text { Measurements } \\
\text { Physical activity (pedometer) } \\
\text { Cardiorespiratory fitness (laboratory } \\
\text { tests: Ruffier-Dickson squat test, } \\
\text { Ebbeling single-stage treadmill walk } \\
\text { test) } \\
\text { Weight status (objectively measured } \\
\text { weight and height) } \\
\text { Blood pressure (measure n.r.) }\end{array}$ & 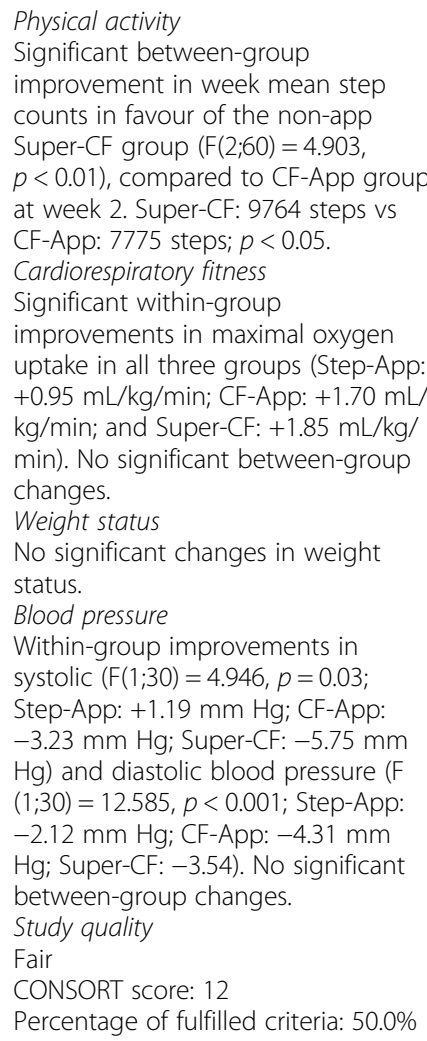 \\
\hline $\begin{array}{l}\text { Safran Naimark et al. } 2015 \\
\text { [58] } \\
\text { Israel }\end{array}$ & $\begin{array}{l}\text { Study design } \\
\text { 2-group RCT } \\
\text { Duration } \\
\text { Intervention exposure: } 14 \text { weeks (2010- } \\
\text { 2011) } \\
\text { Measurement points: baseline, } 14 \text { weeks } \\
\text { Attrition rate: } 14 \% \\
\text { Sample } \\
\text { Adults } \\
N=99 \\
47.9 \text { years/ } \geq 18 \text { years (age range not } \\
\text { reported) } \\
36 \%(M), 64 \%(F)\end{array}$ & $\begin{array}{l}\text { Behaviour change theory } \\
\text { Control systems theory of self-regulation } \\
\text { App features } \\
\text { Newly designed app: } \\
\text { eBalance app that includes goal setting, } \\
\text { self-monitoring of physical activity, } \\
\text { dietary intake, and calorie intake and } \\
\text { expenditure; real-time performance } \\
\text { feedback; information on nutrient intake } \\
\text { compared to dietary recommendations. } \\
\text { Intervention group } \\
\text { Used the app, initial face-to-face } \\
\text { information session on healthy lifestyle }\end{array}$ & $\begin{array}{l}\text { Outcome } \\
\text { Physical activity (minutes/week) } \\
\text { Diet (diet quality score) } \\
\text { Other relevant outcomes } \\
\text { Weight status (body weight in } \\
\text { kilogram, BMI) } \\
\text { BMl } \\
\text { Measures } \\
\text { Physical activity and diet } \\
\text { (questionnaire) } \\
\text { Weight status (objective height and } \\
\text { weight) }\end{array}$ & $\begin{array}{l}\text { Diet } \\
\text { Significant between-group } \\
\text { improvement in diet quality score in } \\
\text { IG at } 14 \text { week follow-up ( }+71 \pm 7.6 \text {; } \\
p<0.001) \text {. } \\
\text { Physical activity } \\
\text { Significant between-group increase } \\
\text { in mean minutes/week of physical } \\
\text { activity in IG at } 14 \text { week follow-up } \\
(+63.0 \pm 20.8 ; p=0.02) \text {. } \\
\text { Weight status }\end{array}$ \\
\hline
\end{tabular}


Table 1 Characteristics of the app-based intervention studies included in the review (Continued)

Random

Silveira et al. 2013 [33]

Van Het Reve et al. 2014 [34]

Switzerland
Control group

Information session on healthy lifestyle only, instructed to continue living a

healthy lifestyle as they understood it

Multi-component versus stand-alone app

intervention

Multi-component

Behaviour change theory

Motivation theory, Transtheoretical

model

App features
Commercially available app:

Intervention exposure: 12 weeks

ActiveLifestyle app that includes

autonomous strength-balance physical

training for independently living older

adults. A strength-balance training plan

with three levels: beginner, intermediate,

and expert. Individual motivation

strategies: positive and negative

reinforcement goal setting self-

Convenience

were randomised but not participants in the control group) monitoring, awareness. Social motivation

strategies: social comparison, monitoring of peers, emotional support, collaboratio with peers to reach common in-game goals. Additional features: a virtual training plan community and communication

features (i.e., private text messaging in the

app, a bulletin board with links to newspapers, videos, and websites) Intervention group

IG 1: Individual group that followed

training using the individual version of

ActiveLifestyle (=individual motivations strategies);

IG 2: Social group that followed training using the social version of the

ActiveLifestyle app (= the individual and social motivation strategies, the virtual training plan community and

communication features)

Control group

Followed exercises with printed

information without additional

motivation strategy.

Multi-component versus stand-alone app

intervention

Stand-alone
Significant between-group decrease for follow-up $(-1.44 \pm 0.40 ; p=0.03)$.

Significant between-group decreas in BMI in IG at 14 week follow-up $\left(-0.48 \mathrm{k} / \mathrm{m}^{2} \pm 0.13 ; p=0.03\right)$

Study quality

High

CONSORT score: 16.5

Percentage of fulfilled criteria: $67.3 \%$

Physical activity

Significant within-group increase in preferred gait speed across al

groups at 12-week follow-up

$(p<.001)$. However, no significant

between-group changes.

Significant within-group and

between-group increases in gait

speed at 12-week follow-up.

Participants walked significantly

faster at post-test $(1.72 \mathrm{~m} / \mathrm{s})$ than at

pre-test $(1.56 \mathrm{~m} / \mathrm{s} ; F=20.1, p<.001$

$c=0.41$ ). The main effect of group

was also significant $(F=5.3, p=.01$

$\zeta=0.27$ ). The individual group

$(1.89 \mathrm{~m} / \mathrm{s})$ was significantly faste

than the control group $(1.45 \mathrm{~m} / \mathrm{s}$;

$\mathrm{t}=3.94, p=.003, \mathrm{~d}=1.31)$, and faster

than the social group $(1.58 \mathrm{~m} / \mathrm{s}$;

$t=2.05, p=.08, d=89$ ), though the

latter did not reach statistical

significance.

Study quality

CONSORT score: 12

Percentage of fulfilled criteria: $48.0 \%$ 
Table 1 Characteristics of the app-based intervention studies included in the review (Continued)

\begin{tabular}{|c|c|c|c|}
\hline $\begin{array}{l}\text { Smith et al. } 2014 \text { [29], Lubans } \\
\text { et al. } 2016 \text { [30] } \\
\text { Australia }\end{array}$ & $\begin{array}{l}\text { Study design } \\
\text { 2-group cluster RCT } \\
\text { Duration } \\
\text { Intervention exposure: } 20 \text { weeks } \\
\text { (Dec } 2012 \text { - June 2013) } \\
\text { Measurement points: baseline, } 8 \text { months } \\
\text { (post-intervention), } 18 \text { months } \\
\text { Attrition rate: } 19 \% \\
\text { Sample } \\
\text { Children } \\
N=361 \\
12.7 \text { years/12-14 years } \\
100 \% \text { (M) } \\
\text { Random }\end{array}$ & $\begin{array}{l}\text { Behaviour change theory } \\
\text { Self-determination theory, social } \\
\text { cognitive theory } \\
\text { App features } \\
\text { Newly designed app: } \\
\text { Goal setting for physical activity and } \\
\text { screen time, self-monitoring (uploading } \\
\text { pedometer measured steps), tailored } \\
\text { motivational and informational messages } \\
\text { via 'push prompts', assessment of } \\
\text { resistance training skill competency, re- } \\
\text { cording fitness challenge results, } \\
\text { resistance training and aerobic exercises. } \\
\text { Intervention group } \\
\text { Goal setting, self-monitoring (steps } \\
\text { through pedometer), fitness challenge } \\
\text { during school sport sessions, teacher } \\
\text { professional development, provision of } \\
\text { fitness equipment to schools, face-to- } \\
\text { face physical activity sessions led by } \\
\text { teachers, lunchtime student mentoring } \\
\text { sessions, researcher-led educational } \\
\text { sessions for children, a smartphone } \\
\text { application and website, parental } \\
\text { education and tips for reducing screen } \\
\text { time through newsletter } \\
\text { Control group } \\
\text { Usual practice (regular school sports and } \\
\text { PE lessons) } \\
\text { Multi-component versus stand-alone app } \\
\text { intervention } \\
\text { Multi-component }\end{array}$ & $\begin{array}{l}\text { Outcome } \\
\text { Physical activity (moderate-to- } \\
\text { vigorous, total; minutes/day) } \\
\text { Sugar-sweetened beverages } \\
\text { consumption (glasses/day) } \\
\text { Other relevant outcomes } \\
\text { Fitness } \\
\text { Weight status (BMl, waist } \\
\text { circumference, body fat) } \\
\text { Measures } \\
\text { Accelerometer (worn on } 7 \\
\text { consecutive days including } \\
\text { weekend) }\end{array}$ \\
\hline
\end{tabular}

Stuckey et al. 2011 [41]

Canada
Study design

Pre-post within-subjects design

Duration

Intervention exposure: 8 weeks

Measurement points: baseline, week 4

week 8

Attrition rate: $8 \%$

Sample

Adults

$N=26$

56.6 years/30-71 years

$25 \%(\mathrm{M}), 75 \%(\mathrm{~F})$
Behaviour change theory

Transtheoretical model

App features

Commercially available app:

Self-monitoring: a Smartphone received

via Bluetooth info from a blood pressure

monitor, a glucometer, and a

pedometer. Weight was manually

entered. Smartphones transmitted self-

monitoring measurements to the

database and allowed participants to

interface with the researchers as well as
Diet

Significant between-group decrease in mean glasses/day of sugar-

sweetened beverage consumption in

IG $(-0.6+0.26 ; p=0.01)$ at 8 -months

follow-up. No significant intervention

effects at 18-months follow-up.

Physical activity

No significant changes in daily MVPA

or overall PA at 8-months and 18-

months follow-ups.

months follow-ups.

Significant between-group difference in mean minutes/day screen-time in favour of IG at 8-months follow-up

$(-30.0 \pm 10.08 ; p=0.03)$ and $18-$

months follow-up $(-32.2 ; 95 \% \mathrm{Cl}$ :

-53.6- $-10.8 ; p=0.03$ ).

Fitness

Significant between-group increase in muscular endurance in IG as measured by mean push-ups repetitions $0.9 \pm 0.49 ; p=0.04)$ and resistance training skills (mean units $5.7 \pm 0.67$ $p<0.001$ ) at 8 -months follow-up.

Intervention effect was sustained for resistance training skills at 18-months follow-up (mean units 5.9, 95\% Cl:

4.5-7.3; $p<0.001)$

Weight status

No significant intervention effects for BMI, waist circumference and

percent of body fat at 8-months and 8-months follow-ups.

Study quality

High

CONSORT score: 21.5

Percentage of fulfilled criteria: $91.5 \%$

Physical activity

Physical activity (steps/day, vo2max) Other relevant outcomes

Weight status (BMI kg/m², waist

Weight status (BM

Blood pressure (diastolic and systolic; $\mathrm{mm} \mathrm{Hg}$ )

Blood glucose

Cholesterol (LDL, HDL, total,

triglycerides; mmol/liter)

Measurements

Physical activity (STEP test)

Significant increase in steps/day in IG at 8-week follow-up $(+1,086 \pm 1613$

$p=0.003)$. Significant increase in

vo2 $\mathrm{max}(\mathrm{ml} / \mathrm{kg} / \mathrm{min})$ at 8 -week

follow-up $(+5.139 \pm 4.911, p<0.001)$. Weight status

Significant reduction in BMI in IG at 8-week follow-up $(-0.465 \pm 0.987$,

$p=0.002$ ).

Blood Glucose

No significant changes. 
Table 1 Characteristics of the app-based intervention studies included in the review (Continued)

\begin{tabular}{|c|c|c|c|c|}
\hline & & $\begin{array}{l}\text { view graphical outputs of their personal } \\
\text { health } \\
\text { indicators. } \\
\text { Intervention group } \\
\text { App intervention plus tailored } \\
\text { counselling (every } 4 \text { weeks) regarding } \\
\text { physical activity and lifestyle } \\
\text { modifications with personal goal setting. } \\
\text { Participants received a stage-matched } \\
\text { activity booklet addressing self-efficacy, } \\
\text { decisional balance, and stage- } \\
\text { appropriate processes of change (a } \\
2 \text { month data plan). } \\
\text { Control group } \\
\text { None } \\
\text { Multi-component versus stand-alone app } \\
\text { intervention } \\
\text { Multi-component }\end{array}$ & $\begin{array}{l}\text { Weight status (objectively measured } \\
\text { weight and height) } \\
\text { Blood pressure } \\
\text { (sphygmomanometer) } \\
\text { Blood glucose and cholesterol } \\
\text { (venepuncture) }\end{array}$ & $\begin{array}{l}\text { Blood pressure } \\
\text { Significant reduction in diastolic } \\
\text { blood pressure in IG at 8-week } \\
\text { follow-up }(-4.375 \pm 5.640, p=0.001) \\
\text { Total cholesterol } \\
\text { Significant reduction in total in } \\
\text { cholesterol levels in IG at 8-week } \\
\text { follow-up (-0.295 } \pm 0.508, p=.009) \text {. } \\
\text { Study quality } \\
\text { Low } \\
\text { CONSORT score: } 11 \\
\text { Percentage of fulfilled criteria: } 45.0 \%\end{array}$ \\
\hline $\begin{array}{l}\text { Van Drongelen et al. } 2014 \\
\text { [54] } \\
\text { The Netherlands }\end{array}$ & $\begin{array}{l}\text { Study design } \\
\text { 2-group RCT } \\
\text { Duration } \\
\text { Intervention exposure: not reported } \\
\text { Measurement points: baseline, } 3 \text { months, } \\
6 \text { months } \\
\text { Attrition rate: } 13.5 \% \\
\text { Sample } \\
\text { Adults } \\
N=502 \\
40.9 \pm 8.4 \text { years/age range: not reported } \\
93 \%(\mathrm{M}), 7 \% \text { (F) } \\
\text { Random }\end{array}$ & $\begin{array}{l}\text { Behaviour change theory } \\
\text { Not reported } \\
\text { App features } \\
\text { Commercially available app: } \\
\text { The MORE Energy app contained } \\
\text { evidence-based advice tailored to flight } \\
\text { schedules and personal characteristics } \\
\text { aiming to reduce fatigue and circadian } \\
\text { disruption as much as possible. } \\
\text { Intervention group } \\
\text { The MORE Energy app + a website } \\
\text { containing more background } \\
\text { information was developed alongside } \\
\text { the smartphone app. } \\
\text { Control group } \\
\text { The participants allocated to the control } \\
\text { group received a minimal intervention } \\
\text { consisting of access to a secure part of } \\
\text { the project website, which contained } \\
\text { basic, non-tailored, fatigue and health- } \\
\text { related information that was already } \\
\text { available within the airline company } \\
\text { (such as information about sleep } \\
\text { hygiene and the working mechanisms } \\
\text { of the biological clock). } \\
\text { Multi-component versus stand-alone app } \\
\text { intervention } \\
\text { Multi-component }\end{array}$ & $\begin{array}{l}\text { Outcome } \\
\text { Physical activity (moderate, vigorous; } \\
\text { days/week) } \\
\text { Diet (breakfast, meal composition, } \\
\text { snacking, hydration, caffeine intake) } \\
\text { Other relevant outcomes } \\
\text { None } \\
\text { Measures } \\
\text { Self-report, questionnaires }\end{array}$ & $\begin{array}{l}\text { Diet } \\
\text { Significant between-group } \\
\text { improvement in snacking behaviour } \\
\text { in IG at } 6 \text {-months follow-up } \\
(\beta=-0.81, p<0.001) \text {. } \\
\text { Physical Activity } \\
\text { Significant between-group increase } \\
\text { in vigorous physical activity in IG at } \\
6 \text {-months follow-up ( } \beta=0.17 \text {, } \\
p=0.028) \\
\text { Study quality } \\
\text { High } \\
\text { CONSORT score: } 17 \\
\text { Percentage of fulfilled criteria: } 68.0 \%\end{array}$ \\
\hline $\begin{array}{l}\text { Walsh et al. } 2016 \text { [55] } \\
\text { Ireland }\end{array}$ & $\begin{array}{l}\text { Study design } \\
\text { 2-group RCT } \\
\text { Duration } \\
\text { Intervention exposure: } 5 \text { weeks }\end{array}$ & $\begin{array}{l}\text { Behaviour change theory } \\
\text { Capability, Opportunity, Motivation, } \\
\text { Behaviour (COM-B) framework, Behavior } \\
\text { Change Wheel }\end{array}$ & $\begin{array}{l}\text { Outcome } \\
\text { Physical activity (steps/day) } \\
\text { Other relevant outcomes } \\
\text { None }\end{array}$ & $\begin{array}{l}\text { Physical activity } \\
\text { Significant between-group } \\
\text { improvements in favour of the app } \\
\text { intervention group ( } F 1,53=4.30 \text {, }\end{array}$ \\
\hline
\end{tabular}

Intervention exposure: 5 weeks
Change Wheel 
Table 1 Characteristics of the app-based intervention studies included in the review (Continued)

\begin{tabular}{|c|c|c|}
\hline & $\begin{array}{l}\text { Measurement points: baseline, } 5 \text { weeks } \\
\text { Attrition rate: } 5 \% \\
\text { Sample } \\
\text { Adults } \\
N=58 \text { ( } 55 \text { analysed) } \\
20.55 \text { years } / 17-26 \text { years } \\
27 \% \text { (M), } 73 \% \text { (F) } \\
\text { Random }\end{array}$ & $\begin{array}{l}\text { App features } \\
\text { Commercially available app: } \\
\text { The 'Accupedo-Pro' pedometer app } \\
\text { includes goal setting, self-monitoring, } \\
\text { performance feedback } \\
\text { Intervention group } \\
\text { Given a walking goal of } 10,000 \text { steps a } \\
\text { day and information related to the } \\
\text { benefits of exercise; instructed to use } \\
\text { the app to achieve and monitor the } \\
\text { goal } \\
\text { Control group } \\
\text { Given a walking goal of } 30 \text { min a day } \\
\text { and information related to the benefits } \\
\text { of exercise } \\
\text { Multi-component versus stand-alone app } \\
\text { intervention } \\
\text { Stand-alone }\end{array}$ \\
\hline $\begin{array}{l}\text { Wharton et al. } 2014 \text { [42] } \\
\text { USA }\end{array}$ & $\begin{array}{l}\text { Study design } \\
\text { 3-group RCT } \\
\text { Duration } \\
\text { Intervention exposure: } 8 \text { weeks } \\
\text { Measurement points: baseline, } 8 \text { weeks } \\
\text { Attrition rate: } 18 \% \\
\text { Sample } \\
\text { Adults } \\
N=57 \text { ( } 47 \text { analysed) } \\
42.0 \text { years } / 18-65 \text { years } \\
26 \%(\mathrm{M}), 74 \% \text { (F) } \\
\text { Random (but controlled for sex, age and } \\
\text { BMI) }\end{array}$ & $\begin{array}{l}\text { Behaviour change theory } \\
\text { Not reported } \\
\text { App features } \\
\text { Commercially available app: } \\
\text { The 'Lose It!' app includes self-monitoring } \\
\text { of dietary intake; performance feedback } \\
\text { via daily calorie gauge graphic, calculated } \\
\text { energy allotment and individual } \\
\text { anthropometric data } \\
\text { Intervention group } 1 \\
\text { Goal setting for weight loss, app group } \\
\text { self-monitored dietary intake via an app } \\
\text { diary (Lose It!), instruction to expend } 150 \\
\text { calories/day via structured exercise, } \\
\text { received a chart of physical activity } \\
\text { options with approx. energy expenditures } \\
\text { for } 30,40,50 \text { and } 60 \text { min. } \\
\text { Intervention group } 2 \\
\text { Goal setting for weight loss, personally } \\
\text { written diet plan, memo group self- } \\
\text { monitored dietary intake via the memo } \\
\text { function of their smartphone, face-to- } \\
\text { face nutrition counselling sessions prior } \\
\text { to the start of study, weekly emails to } \\
\text { encourage healthy eating, instruction to } \\
\text { expend } 150 \text { calories/day via structured } \\
\text { exercise, received a chart of PA options } \\
\text { with approx. energy expenditures for } 30 \text {, } \\
40,50 \text { and } 60 \text { min } \\
\text { Intervention group } 3 \\
\text { Goal setting for weight loss, personally } \\
\text { written diet plan, self-monitoring of } \\
\text { dietary intake via paper and pencil }\end{array}$ \\
\hline
\end{tabular}

\section{Measures}

Physical activity (Accupedo-Pro

pedometer app)

Outcome

Diet

Other relevant outcomes

Weight status (weight in pounds, BMI)

Measures

Diet (self-report, questionnaire:

Healthy Eating Index)

Weight status (questionnaire)
$P=0.043, \mathrm{np} 2=0.08$ ): significantly higher increase in steps in app intervention group (2393) compared to control group $(1101 ; \mathrm{t} 53=2.07$, $p=0.043$. Significant within-group improvements for both intervention group (t27 $=-6.14, p<.001)$ and control group (t26 $=-2.25, p=.033$ ). Study quality

CONSORT score: 12

Percentage of fulfilled criteria: 50.0\%

Diet

No significant change in dietary

intake.

Weight status

No significant difference in betweengroup change in weight, but

significant within-group decrease in body weight in all groups (IG1: -3.5 \pm 1.0, IG2: $-6.5 \pm 1.4$, IG3: $-4.4 \pm 1.2$ mean pounds).

No significant change in BMI.

Study quality

CONSORT score: 10.5

Percentage of fulfilled criteria: $43.0 \%$ 
Table 1 Characteristics of the app-based intervention studies included in the review (Continued)

\begin{tabular}{|c|c|c|c|c|}
\hline & & $\begin{array}{l}\text { notebook, personally written diet plan, } \\
\text { face-to-face nutrition counselling } \\
\text { sessions prior to the start of study, } \\
\text { weekly emails to encourage healthy } \\
\text { eating, instruction to expend } 150 \text { calories/ } \\
\text { day via structured exercise, received a } \\
\text { chart of PA options with approx. energy } \\
\text { expenditures for } 30,40,50 \text { and } 60 \text { min } \\
\text { Multi-component versus stand-alone app } \\
\text { intervention } \\
\text { Stand-alone }\end{array}$ & & \\
\hline $\begin{array}{l}\text { Wang et al. } 2015 \text { [43] } \\
\text { USA }\end{array}$ & $\begin{array}{l}\text { Study design } \\
\text { 2-group RCT } \\
\text { Duration } \\
\text { Intervention exposure: } 6 \text { weeks } \\
\text { Measurement points: baseline, weeks 1, } \\
\text { 2, 3, 4, 5, and } 6 \text { (Fitbit), } 6 \text { weeks } \\
\text { (accelerometer) } \\
\text { Attrition rate: } 9 \% \\
\text { Sample } \\
\text { Adults } \\
N=67 \\
49.3 \text { years/18-69 years } \\
9 \%(M), 91 \% \text { (F) } \\
\text { Random }\end{array}$ & $\begin{array}{l}\text { Behaviour change theory } \\
\text { Not reported } \\
\text { App features } \\
\text { Commercially available app: } \\
\text { The Fitbit One Tracker that include self- } \\
\text { monitoring through a wearable tracker } \\
\text { and website/mobile app. } \\
\text { Intervention group } \\
\text { Daily SMS-based physical activity } \\
\text { prompts plus self-monitoring with the } \\
\text { FitBit One } \\
\text { Control group } \\
\text { Self-monitoring with the FitBit One } \\
\text { Multi-component versus stand-alone } \\
\text { app intervention } \\
\text { Multi-component }\end{array}$ & $\begin{array}{l}\text { Outcomes } \\
\text { Physical activity (moderate-to- } \\
\text { vigorous, total; steps/day and } \\
\text { minutes/week) } \\
\text { Measures } \\
\text { Physical activity (accelerometer, } \\
\text { Fitbit) }\end{array}$ & $\begin{array}{l}\text { Physical activity } \\
\text { Significant within-group increase in } \\
\text { physical activity in IG at 1-week } \\
\text { follow-up (steps/day: +1,266, SE: 491, } \\
p=0.01 ; \text { moderate-to-vigorous phys- } \\
\text { ical activity minutes/week: }+17.8, \text { SE: } \\
8.5, p=0.04 \text {; total physical activity: } \\
+38.3, \text { SE: } 15.9, p=0.02 \text { ). } \\
\text { Significant within-group increase in } \\
\text { moderate-to-vigorous physical } \\
\text { activity minutes/week in CG (4.3; SE: } \\
\text { 2.0; } p=0.04 \text { ) at } 6 \text {-week follow-up. } \\
\text { However, the significant within- } \\
\text { group changes were not maintained } \\
\text { at the weeks } 2-6 \text { follow-ups. } \\
\text { Moreover, no significant between- } \\
\text { group changes in steps, as well as } \\
\text { moderate-to-vigorous and total } \\
\text { physical activity at 6-week follow-up. } \\
\text { Study quality } \\
\text { High } \\
\text { CONSORT score: } 17.5 \\
\text { Percentage of fulfilled criteria: } 71.4 \%\end{array}$ \\
\hline
\end{tabular}




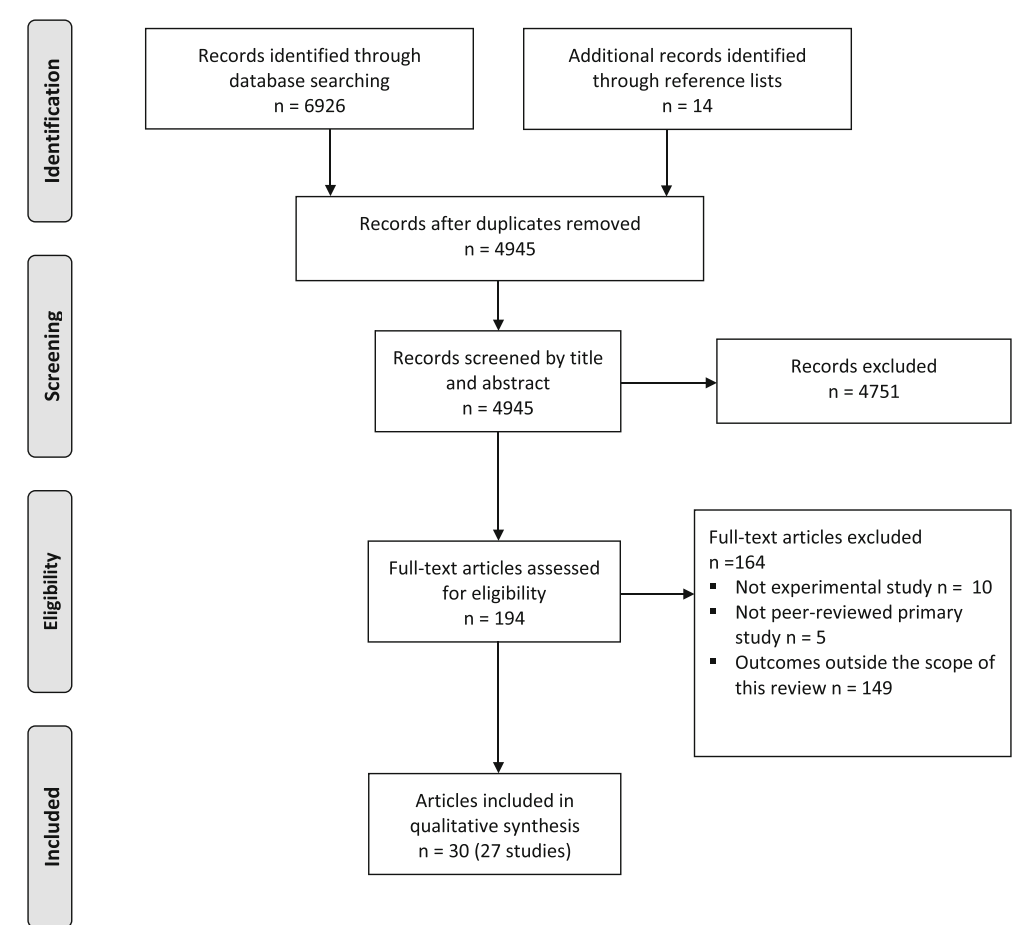

Fig. 1 Flowchart of study selection process

Twenty-three studies [31-36, 38, 39, 41-46, 48-58] targeted adults (mean age: 41.5 years, range: $18-71$ years) and four studies $[29,37,40,47]$ targeted children or adolescents (mean age: 12.4 years, range: $8-17$ years). The total number of participants across the 27 studies was 2699 (510 children/adolescents, 2189 adults). Samples sizes ranged from 17 to 502 (mean sample size: 100). The duration of interventions ranged from 1-24 weeks, with an average intervention duration of 10 weeks. Follow-up assessments were at 4 weeks $(n=5), 8$ weeks $(n=8)$, 12 weeks $(n=9), 20$ weeks $(n=2), 6$ months $(n=2)$, 9 months $(n=1)$ and 18 months $(n=1)$. Attrition rates ranged from $0 \%$ [36] to 57\% [51], with an average attrition rate of $17 \%$. The majority of studies $[31,33,35-45,47,48$, $50,53,55-58]$ reported higher rates of female participation (on average $64 \%$ of participants were female). The targeted health behaviours were dietary intake $(n=13)$, physical activity $(n=21)$ and sedentary behaviour $(n=5)$. Other reported lifestyle-related health outcomes were: weight status $(n=11)$; fitness $(n=3)$; blood pressure $(n=4)$; blood glucose $(n=1)$; cholesterol $(n=1)$; and quality of life $(n=2)$. Ten studies $[33,36-38,43-45$, $49,50,55,57]$ targeted a single health behaviour, whilst 17 studies $[29,31,35,39-42,46-48,50-56,58]$ targeted multiple health behaviours and related health outcomes. Fourteen studies [33, 37-40, 42, 45-47, 51, 52, 55-57] involved interventions delivered solely via an app (stand-alone intervention) and 13 studies $[29,31,35,36$,
$41,43,44,48-50,53,54,58]$ involved interventions that used apps in conjunction with other intervention strategies (multi-component intervention), such as physical education, parental education, counselling sessions, printed materials, motivational emails, websites and pedometer use. Fifteen studies $[29,31,36-39,45,46$, $48-50,52,56-58]$ used a newly designed app in the intervention and 12 studies [33, 35, 40-44, 47, 51, 53-55] used a commercially available app. Further, 15 of the 27 studies reported intervention designs based on behaviour change theories, such as Self-determination Theory $(n=3)$ [29, 35, 37], Transtheoretical Model $(n=4)$ $[31,33,41,48]$, Social Cognitive Theory $(n=4)[29,39,44$, 46], Theory of Planned Behaviour $(n=2)[45,50]$, Control Systems Theory of Self-regulation $(n=1)$ [58], and the Behaviour Change Wheel $(n=1)$ [55].

\section{Study quality}

A detailed summary of quality assessments of included studies is presented in the Additional file 3. Overall, study quality ranged from high $(n=11)[29,31,43-45$, $47,48,50,53,54,58]$, to fair $(n=8)[35,37,46,49,55-$ 57], and low $(n=8)[33,36,38,39,41,42,51,52]$. Study quality of interventions targeted to children/adolescents was high $(n=2)$ [29, 47] and fair $(n=2)$ [37, 40], and study quality of interventions targeted to adults ranged from high $(n=9)[31,44,45,48,50,53,54,58]$, to fair $(n=6)$ [35, 46, 49, 55-57], and low $(n=8)$ [33, 36, 38, 
39, 41, 42, 51, 52]. Most of the 13 interventions that used an app in combination with other intervention strategies were of high quality $(n=9)[29,31,43,44,48$, $50,53,54,58]$, whilst most of the 14 stand-alone app interventions were of fair $(n=6)$ [37, 40, 46, 55-57] or low quality $(n=6)[33,38,39,42,51,52]$. Study quality did not differ markedly between app interventions targeting multiple health behaviours and related health outcomes (high: $n=8$, fair: $n=4$, low: $n=5$ ) and those targeting a single health behaviour (high: $n=3$, fair: $n=4$, low: $n=3)$. On average, the included studies fulfilled $61 \%$ of the assessment criteria (range: 34-92\%). Most studies met the CONSORT requirements to provide a strong scientific rationale and described their participant eligibility, statistical methods and interventions clearly. Fewer studies reported sample size calculations $[29,31,35,43,47,50,53-55,57,58]$ and included randomisation $[29,31,35,37,39,43-48,50$, $52-55,58]$ and blinding procedures [31, 44-50, 53] in their study design. Attrition rates were reported or could be calculated for the majority of studies [29, 31, 33, 35-51, 53-58].

\section{Intervention efficacy}

A summary of intervention effects for the included lifestyle behaviour outcomes (diet, physical activity, sedentary behaviour) and related health outcomes (weight status, fitness, blood pressure, glucose, cholesterol, quality of life) are presented in Table 2. Overall, a slightly larger proportion of single health behaviour interventions ( 5 out of 10; $50 \%)[33,45,49,55,57]$ showed significant betweengroup improvements than multiple health behaviour interventions (7 out of 17 ; 41\%) [29, 31, 48, 50, 53, 54, 58]. Further, a larger proportion of interventions that used an app in conjunction with other intervention strategies ( 8 out of 13; 62\%) demonstrated significant between-group improvements in the behavioural and health outcomes $[29,31,48-50,53,54,58]$ compared to stand-alone app interventions (5 out of $14 ; 36 \%$ ) [33, 40, 45, 55, 57].

\section{Children and adolescents}

Of the four studies that specifically targeted children and/or adolescents, one study [29] reported significant between-group improvements in diet, sedentary behaviour and fitness in the app intervention group. Another study [37] reported a significant within-group increase in physical activity, but no significant difference between groups. The remaining two studies [40,47] reported no significant changes in the behavioural or related health outcomes.

\section{Adults}

Of the 23 studies that targeted adults, 17 studies reported significant improvements in $\operatorname{diet}(n=6)[31,38$,
$45,54,57,58]$, physical activity $(n=13)[31,33,36,37$, $39,41,43,48-50,53-55,58]$, sedentary behaviour $(n=1)$ [39], and other improved outcomes including weight status $(n=4)[31,41,42,58], 49]$, fitness $(n=1)$ [56], blood pressure $(n=2)$ [41, 56] and cholesterol $(n=1)$ [41]. Of the studies reporting significant findings, 11 studies detected significant between-group differences in diet $(n=5)$ $[31,45,54,57,58]$, physical activity $(n=9)[31,33,48-50$, $53-55,58]$ and weight status $(n=2)[31,58]$ in favour of the app intervention group. Seven studies found significant within-group improvements in diet $(n=1)$ [38], physical activity $(n=4)$ [33, 36, 37, 39, 41, 43], sedentary behaviour $(n=1)$ [39] and weight status $(n=2)$ $[41,42,56]$, blood pressure $(n=2)[41,56]$ and cholesterol levels $(n=1)$ [41]. Five studies [35, 44, 46, 51, 52] reported no significant changes in the health outcomes of interest, and no significant findings were found in relation to the outcome glucose levels (assessed in one study).

\section{Characteristics of efficacious interventions}

App interventions showing significant between-group improvements in the behavioural and health outcomes tended to be multi-component interventions [31, 36, 41, $43,48-50,53,54,58]$, with sample sizes above 90 participants $[35,43,44,47-49,53]$ and intervention durations longer than 8 weeks [37, 42-45, 47, 49]. A slightly larger proportion of single health behaviour [33, 45, 49, 55, 57] versus multiple health behaviour interventions [29, 31, $48,50,53,54,58$ ] demonstrated between-group improvements $(50 \%$ versus $41 \%$, respectively). Further, most of the interventions $[29,31,33,39,42,45,48-$ $50,53,55,56,58]$ showing significant improvements in the behavioural and health outcomes included goal-setting, self-monitoring and performance feedback in the app design. Some efficacious interventions also incorporated other behaviour change techniques, such as motivational messages [29, 36, 57], health education/tailored advice [29, 31, 38, 48, 54, 57], reinforcement $[33,39,40,45]$, gamification in the form of exergames, award and rewards [37-40, 50], social support through interaction with peers $[33,37$, 50] and friendly team challenges [29, 37, 39, 45]. There was not enough data to identify which behaviour change techniques determined intervention efficacy. Moreover, there was no difference in the behaviour change techniques incorporated in apps for children compared to those used in apps for adults. Eleven studies $[31,38,40,43,45,48-50,54,56-58]$ out of the 19 studies showing significant improvements in behavioural and health outcomes reported usage statistics to determine participants' engagement with the app. Three of these studies [38, 43, 49] examined associations between app usage and changes in the behavioural and health outcomes. Their findings showed that 
Table 2 Summary of intervention effects on behaviour outcomes and related health outcomes

\begin{tabular}{|c|c|c|c|c|c|c|c|c|c|}
\hline \multirow[b]{2}{*}{ Study } & \multicolumn{3}{|c|}{ Behaviour outcomes } & \multicolumn{6}{|c|}{ Related health outcomes } \\
\hline & Diet & $\begin{array}{l}\text { Physical } \\
\text { activity }\end{array}$ & $\begin{array}{l}\text { Sedentary } \\
\text { behaviour }\end{array}$ & $\begin{array}{l}\text { Weight } \\
\text { status }\end{array}$ & Fitness & $\begin{array}{l}\text { Blood } \\
\text { pressure }\end{array}$ & Glucose & Cholesterol & $\begin{array}{l}\text { Quality of } \\
\text { life }\end{array}$ \\
\hline \multicolumn{10}{|l|}{ Children } \\
\hline Direito et al. 2015 [47] & & 0 & 0 & & 0 & & & & \\
\hline Garde et al. 2015 [37] & & $+(w)$ & & & & & & & \\
\hline Nollen et al. 2014 [40] & 0 & & 0 & 0 & & & & & \\
\hline $\begin{array}{l}\text { Smith et al. } 2014 \text { [29], } \\
\text { Lubans et al. } 2016 \text { [30] }\end{array}$ & $+(b)$ & 0 & $+(\mathrm{b})$ & 0 & $+(b)$ & & & & \\
\hline \multicolumn{10}{|l|}{ Adults } \\
\hline Choi et al. 2016 [44] & & 0 & & & & & & & \\
\hline Cowdery et al. 2015 [35] & & 0 & & 0 & & 0 & & & \\
\hline Elbert et al. 2016 [57] & $+(\mathrm{b})$ & & & & & & & & \\
\hline Fukuoka et al. 2010 [36] & & $+(W)$ & & & & & & & \\
\hline Gasser et al. 2006 [52] & 0 & 0 & & 0 & & & & & \\
\hline Gilliland et al. 2015 [38] & $+(w)$ & & & & & & & & \\
\hline Gilson et al. 2016 [51] & 0 & 0 & & & & & & & \\
\hline Glynn et al. 2014 [53] & & $+(b)$ & & 0 & & 0 & & & 0 \\
\hline Hebden et al. 2014 [48] & 0 & $+(b)$ & 0 & 0 & & & & & \\
\hline King et al. 2013 [39] & & $+(w)$ & $+(w)$ & & & & & & \\
\hline Kirwan et al. 2012 [49] & & $+(b)$ & & & & & & & \\
\hline Maher et al. 2015 [50] & & $+(b)$ & & & & & & & 0 \\
\hline Mummah et al. 2016 [45] & $+(b)$ & & & & & & & & \\
\hline $\begin{array}{l}\text { Partridge et al. } 2015 \text { [31], } \\
\text { Allman-Farinelli et al. } 2016 \text { [32] }\end{array}$ & $+(b)$ & $+(b)$ & & $+(b)$ & & & & & \\
\hline Rabbi et al. 2015 [46] & 0 & 0 & & & & & & & \\
\hline Rospo et al. 2016 [56] & & $-(b)$ & & & $+(w)$ & $+(w)$ & & & \\
\hline Safran Naimark et al. 2015 [58] & $+(b)$ & $+(b)$ & & $+(b)$ & & & & & \\
\hline $\begin{array}{l}\text { Silveira et al. } 2013 \text { [33], } \\
\text { Van Het Reve et al. } 2014 \text { [34] }\end{array}$ & & $+(b)$ & & & & & & & \\
\hline Stuckey et al. 2011 [41] & & $+(w)$ & & $+(w)$ & & $+(w)$ & 0 & $+(w)$ & \\
\hline Van Drongelen et al. 2014 [54] & $+(b)$ & $+(b)$ & & & & & & & \\
\hline Wharton et al. 2014 [42] & 0 & & & $+(w)$ & & & & & \\
\hline Walsh et al. 2016 [55] & & $+(b)$ & & & & & & & \\
\hline Wang et al. 2015 [43] & & $+(w)$ & & & & & & & \\
\hline
\end{tabular}

+ (b): between-group significant improvements in favour of app intervention group, - (b): between-group significant improvements in favour of non-app control group, $+(w)$ : within-group significant improvement, 0 : no significant change

higher app usage was associated with improvements in physical activity and healthy eating [38, 43, 49].

\section{Discussion}

This systematic review found modest evidence for the efficacy of app interventions to improve diet, physical activity and sedentary behaviours for NCD prevention. Overall, 19 out of the 27 identified studies reported significant improvements in behavioural and related health outcomes. Most of these studies reported significant between-group improvements in the app intervention group versus comparison group, which is considered the gold standard to demonstrate intervention efficacy [59]. Notwithstanding study limitations, the findings from this review indicate that apps can be an effective tool to improve health behaviours. The advantages of smartphone apps over other intervention delivery modes such as websites, face-to-face counselling and group sessions may partially explain the efficacy of app interventions. Given that many people have busy lifestyles, they value 
convenient access to health behaviour change programs that provide information and advice, real-time selfmonitoring, feedback, reinforcement, social support, and rewards 'on the go' [60]. The appeal of smartphones for assistance in health promotion concurs with the trend that more people are seeking health information via mobile devices [61, 62]. In this context, apps provide the opportunity to bring behavioural interventions into real life situations where people make decisions about their health.

Despite the potential of apps, half of the interventions identified in this review used apps in conjunction with other intervention strategies. The remaining interventions were stand-alone interventions where the app was the sole intervention component. Importantly, however, is the observation that most of the multi-component interventions demonstrated significant between-group improvements in behavioural and health outcomes, whereas fewer stand-alone app interventions reported significant between-group improvements. This raises the question whether multi-component interventions yield stronger intervention effects than stand-alone app interventions. Many reviews of health behaviour change interventions [63-67] recommend the use of multiple intervention strategies to achieve long-term health behaviour change. Reviews of website-delivered interventions $[9,28,68]$ have also shown stronger behaviour change effects when combined with other intervention strategies such as SMS, telephone coaching and motivational emails. As such, it is likely that the integration of apps in multi-component interventions produces stronger health outcomes compared to stand-alone app interventions; however, this remains to be tested in future trials.

The majority of app interventions were targeted to adults; only four out of the 27 identified app interventions were specifically aimed at children or adolescents. This is consistent with previous e \& mHealth reviews $[11,12,25,69]$ showing that internet and mobile phone delivered interventions have mainly targeted adult populations. Although smartphones and tablets have become an integral part of children's lives [70], surprisingly few interventions have yet utilised app technology in pediatric health behaviour change programs [12]. However, as exergame and serious game apps are becoming increasingly popular among children and adolescents [71], it is likely that more app studies focussing on children will be conducted in the near future.

The average attrition rate (i.e., participant loss to follow-up) reported in the app interventions was $17 \%$, which is lower compared average attrition rates of 23$27 \%$ found in web-based interventions [28, 68, 72]. Lower participant attrition indicates less bias in the estimated intervention effects [73]. However, participant attrition does not capture participants' engagement with the app intervention which also determines intervention effects. For example, web- and app-based interventions that have examined participant engagement found that higher levels of website and app usage were associated with increased intervention efficacy [38, 43, 68]. Despite this, less than half of the studies reported usage statistics to determine participants' engagement with the app. This is consistent with previous reviews $[24,28,68]$ reporting that few internet and mobile interventions recorded participants' engagement with the intervention technologies. Given the relative ease by which app usage statistics can be tracked, it is unfortunate that this data is not being collected and published for all studies in this area. We know that participant engagement, measured by number of website logins, usually declines after the first few weeks in website and social media interventions [24, 28, 68]. An example is the Australian 10,000 Steps program, a successful, freely available web- and appbased intervention to promote physical activity [61]. Participants' engagement with the 10,000 Steps website and app lasts on average 5-6 weeks, with longer usage duration (on average 8 weeks) being observed in people who use both the 10,000 Steps website and app [61] This rapid decline in usage concurs with qualitative research showing that people often lack commitment to using any particular app and they tend to engage in only transient, casual app use [60]. Since participant engagement determines intervention exposure, and level of intervention exposure determines intervention efficacy $[28,61,74]$, better understanding of factors that improve participant engagement and retention is needed.

To increase user engagement with health behaviour change apps, more information is needed about what app features and behaviour change techniques people value and use. Recent focus group data showed that $[60,75]$ that young people value health behaviour apps that require low effort, are pleasant to use, are developed by credential experts, enable self-monitoring, provide advice on how to change behaviour, include positively framed alerts/reminders (but not too frequent), provide accurate tracking functions, incorporate adequate privacy settings, and clearly show what the app will do (no surprises). Some of these user preferences were part of the efficacious apps identified in this review. For example, most apps included goal-setting, self-monitoring and performance feedback $[29,31,33,39,42,45,48-50,53,55$, $56,58]$. Additionally, some apps incorporated tailored advice, motivational prompts and reinforcement, gamification, social support or friendly team challenges [29, 33, $37-40,45,48,50,54,57]$. Reviews of web-based interventions have demonstrated that interventions including more behaviour change techniques are more effective $[74,76]$. This may also apply to app-based interventions. 
However, it remains unclear what the optimal number and combination of app features and behaviour change techniques is to increase user retention and ultimately intervention efficacy. It is also possible that efficacy declines when too many features or techniques are implemented. Furthermore, socio-demographic factors (e.g., sex, age, education) and psychosocial factors (e.g., attitudes, perceived benefits, enjoyment) may also influence app usage [60]. Emerging research on the adoption of app technology showed that higher app usage is associated with being female and of younger age [61,77], as well as with personal interest in new technologies, positive attitudes towards smartphone apps and perceived benefit of use [77]. Therefore, targeting and tailoring smartphones apps to specific population groups may also enhance the efficacy of app-based interventions.

\section{Strengths and limitations}

Strengths of this systematic review are that it was conducted and reported according to PRISMA guidelines [23], and study quality was systematically assessed using the CONSORT checklist [26]. The search strategy was comprehensive, and study selection, data extraction, and quality rating were completed by two independent reviewers, as is standard practice for high quality systematic review [23]. These procedures ensure accuracy of the reviewed data. The scope of this review was limited to app interventions improving behavioural and related health outcomes for prevention; hence app interventions relating to chronic disease treatment were not captured in this review. Few app interventions identified in this review focused on dietary and sedentary behaviours which makes it more difficult to draw conclusions on the efficacy of app interventions targeting these behaviours, as opposed to those targeting physical activity behaviour. Furthermore, the included studies varied widely in terms of methodological quality, with some studies scoring very poorly, thereby reducing the trust that can be placed in their findings. Finally, the possibility of publication bias should also be acknowledged. As with all systematic reviews examining the efficacy of interventions, it is possible that some studies with null findings have not been published [78].

\section{Recommendations for future research}

Based on this review, it is recommended that future studies:

- Test the efficacy of specific app features and behaviour change techniques in high quality controlled trials to distinguish effective from ineffective intervention components.

- Directly compare the efficacy of stand-alone app intervention compared to multi-component interventions that use apps in combination with other intervention strategies.

- Compare the efficacy of app interventions to other intervention delivery modes, such as website, printbased and face-to-face interventions.

- Utilise larger sample sizes to ensure they are sufficiently powered to detect significant intervention effects.

- Tailor app interventions to specific population groups (e.g., women, young people) in whom usage and adoption of app technology is high.

- Report app usage statistics using objective and self-report measures to examine levels of and reasons for participant (dis)engagement and intervention exposure.

- Explore the optimal duration and intensity of app interventions to ensure user engagement and retention as the intervention progresses.

- Identify factors that increase user engagement and retention in app interventions to sustain behavioural health improvments in the long-term.

- Investigate the relationship between user engagement and intervention efficacy, whilst taking into account socio-demographic and psychosocial factors.

\section{Conclusions}

Interventions using apps to improve diet, physical activity and sedentary behaviour for prevention show promise for effective behaviour change in children and adults. The evidence base is largest for the use of apps to increase physical activity in adults. Fewer interventions have used apps to improve dietary and sedentary behaviours, and very few app interventions have targeted children and adolescents. Multi-component interventions that combine apps with other intervention strategies appear to be more effective than stand-alone app interventions, however, this remains to be tested further in controlled trials. Overall, there is still considerable scope to improve the efficacy of app-based interventions. In particular, intervention studies should gather more app usage statistics to identify factors that improve user engagement and retention, and its relationship with intervention efficacy. In addition, more formative research is needed to determine the optimal number and combination of app features, behaviour change techniques, and level of participant contact needed to maximise user engagement and ultimately intervention efficacy.

\section{Additional files}

Additional file 1: Completed PRISMA checklist. (DOCX $77 \mathrm{~kb})$

Additional file 2: Search strategy used in the databases. (DOCX $32 \mathrm{~kb}$ )

Additional file 3: Quality assessment for all included studies. (DOCX $20 \mathrm{~kb}$ ) 


\section{Abbreviations}

Apps: Applications; CONSORT: Consolidated standards of reporting trials; NCDs: Non-communicable diseases; PRISMA: Preferred reporting items for systematic reviews and meta-analyses

\section{Acknowledgements}

SS is supported by an Early Career Fellowship (GNT1125586) from the Australian National Health and Medical Research Council. CV (ID 100427) and MJD (ID 100029) are supported by Future Leader Fellowships from the National Heart Foundation of Australia.

\section{Funding}

SS is supported by an Early Career Fellowship (GNT1125586) from the Australian National Health and Medical Research Council. CV (ID 100427) and MJD (ID 100029) are supported by Future Leader Fellowships from the National Heart Foundation of Australia.

\section{Availability of data and materials}

Not applicable.

\section{Authors' contributions}

SS, SA, WVL and CV conceptualised and designed the study. SS, SA, WVL and $\mathrm{NAB}$ conducted the search, article selection, data extraction and quality assessment. SS drafted and wrote the manuscript. SA, WVL, CV, MJD, SLW and $N A B$ were involved in interpretation of data and critically revising the manuscript. All authors read and approved the final manuscript.

\section{Competing interests}

The authors declare that they have no competing interests.

\section{Consent for publication}

Not applicable.

\section{Ethics approval and consent to participate}

\section{Not applicable.}

\section{Author details}

${ }^{1}$ Central Queensland University, School of Health, Medical and Applied Sciences, Physical Activity Research Group, Building 77, Bruce Highway, Rockhampton QLD 4702, Australia. ${ }^{2}$ Ghent University, Department of Public Health, De Pintelaan 185-4 K3 room 036, 9000 Ghent, Belgium. ${ }^{3}$ Central Queensland University, School of Health, Medical and Applied Sciences, Building 6, Bruce Highway, Rockhampton QLD 4702, Australia. ${ }^{4} T$ The University of Newcastle, School of Medicine \& Public Health, Priority Research Centre for Physical Activity and Nutrition, University Drive, Callaghan NSW 2308, Australia.

Received: 6 July 2016 Accepted: 24 November 2016 Published online: 07 December 2016

\section{References}

1. World Health Organization. Global status report on noncommunicable diseases. World Health Organization. 2014. http://www.who.int/nmh/ publications/ncd-status-report-2014/en/. Accessed 5 Jul 2016.

2. Biddle SJ, Pearson N, Ross GM, Braithwaite R. Tracking of sedentary behaviours of young people: a systematic review. Prev Med. 2010;51:345-51.

3. Craigie AM, Lake AA, Kelly SA, Adamson AJ, Mathers JC. Tracking of obesityrelated behaviours from childhood to adulthood: a systematic review. Maturitas. 2011:70:266-84.

4. Statistica. Number of smartphone users worldwide from 2014 to 2019 Statistica. 2016. http://www.statista.com/statistics/330695/number-ofsmartphone-users-worldwide/. Accessed 5 Jul 2016.

5. Australian Communications and Media Authority. Communications report 2014-15. Australian communications and media authority. 2015. http:// www.acma.gov.au/theACMA/Library/Corporate-library/Corporatepublications/communications-report-2014-15. Accessed 5 July 2016.

6. Group Special Mobile Association. The mobile economy. Group special mobile association. 2015. http://www.gsmamobileeconomy.com/GSMA_ Global_Mobile_Economy_Report_2015.pdf. Accessed 5 July 2016.
7. Middelweerd A, Mollee JS, van der Wal CN, Brug J, te Velde SJ. Apps to promote physical activity among adults: a review and content analysis. Int $J$ Behav Nutr Phys Act. 2014;11:97.

8. Direito A, Pfaeffli Dale L, Shields E, Dobson R, Whittaker R, Maddison R. Do physical activity and dietary smartphone applications incorporate evidencebased behaviour change techniques? BMC Public Health. 2014;14:646.

9. Hutchesson MJ, Rollo ME, Callister R, Collins CE. Self-monitoring of dietary intake by young women: Online food records completed on computer or smartphone are as accurate as paper-based food records but more acceptable. J Acad Nutr Diet. 2015;115:87-94.

10. Fanning J, Mullen SP, McAuley E. Increasing physical activity with mobile devices: a meta-analysis. J Med Internet Res. 2012;14:61-71.

11. Monroe CM, Thompson DL, Bassett DR, Fitzhugh EC, Raynor HA. Usability of mobile phones in physical activity-related research: a systematic review. Am J Health Educ. 2015:46:196-206.

12. Bort-Roig J, Gilson ND, Puig-Ribera A, Contreras RS, Trost SG. Measuring and influencing physical activity with smartphone technology: a systematic review. Sports Med. 2014;44:671-86.

13. Zhao J, Freeman B, Li M. Can mobile phone apps influence people's health behavior change? An evidence review. J Med Internet Res. 2016;18:e287.

14. Lau PW, Lau EY, Wong del P, Ransdell L. A systematic review of information and communication technology-based interventions for promoting physical activity behavior change in children and adolescents. J Med Internet Res. 2011;13:e48.

15. Schoffman DE, Turner-McGrievy G, Jones SJ, Wilcox S. Mobile apps for pediatric obesity prevention and treatment, healthy eating, and physical activity promotion: Just fun and games? Transl Behav Med. 2016;3:320-5.

16. Conroy DE, Yang $\mathrm{CH}$, Maher JP. Behavior change techniques in top-ranked mobile apps for physical activity. Am J Prev Med. 2014;46:649-52.

17. Sharp DB, Allman-Farinelli M. Feasibility and validity of mobile phones to assess dietary intake. Nutr. 2014;30:1257-66.

18. Payne HE, Lister C, West JH, Bernhardt JM. Behavioral functionality of mobile apps in health interventions: a systematic review of the literature. JMIR Mhealth Uhealth. 2015;3:e20.

19. Wang J, Wang Y, Wei C, Yao NA, Yuan A, Shan Y, et al. Smartphone interventions for long-term health management of chronic diseases: an integrative review. Telemed J E Health. 2014;20:570-83.

20. Woo J, Chen J, Ghanavati V, Lam R, Mundy N, Li LC. Effectiveness of cellular phone-based interventions for weight loss in overweight and obese adults: a systematic review. Orthop Muscul Syst. 2013:3:141.

21. Okorodudu DE, Bosworth HB, Corsino L. Innovative interventions to promote behavioral change in overweight or obese individuals: a review of the literature. Ann Med. 2015;47:179-85.

22. Bardus M, van Beurden SB, Smith JR, Abraham C. A review and content analysis of engagement, functionality, aesthetics, information quality, and change techniques in the most popular commercial apps for weight management. Int J Behav Nutr Phys Act. 2016;13:35.

23. Moher D, Liberati A, Tetzlaff J, Altman DG, Group TP. Preferred reporting items for systematic reviews and meta-analyses: the PRISMA statement. PLoS Med. 2009;6:e1000097.

24. Maher CA, Lewis LK, Ferrar K, Marshall S, De Bourdeaudhuij I, Vandelanotte C. Are health behavior change interventions that use online social networks effective? A systematic review JMIR. 2014;16:e40.

25. Stephens J, Allen J. Mobile phone interventions to increase physical activity and reduce weight: a systematic review. J Cardiovasc Nurs 2013;28:320-9.

26. Moher D, Hopewell S, Schulz KF, Montori V, Gøtzsche PC, Deveraux PJ, et al. CONSORT 2010 explanation and elaboration: updated guidelines for reporting parallel group randomised trials. BMJ. 2010;340:c869.

27. Schoeppe S, Duncan MJ, Badland H, Oliver M, Curtis C. Associations of children's independent mobility and active travel with physical activity, sedentary behaviour and weight status: a systematic review. JSAMS. 2013;16:312-9.

28. Davies C, Spence JC, Vandelanotte C, Caperchione CM, Mummery WK Meta-analysis of internet-delivered interventions to increase physical activity levels. Int J Behav Nutr Physl Act. 2012:9:52.

29. Smith JJ, Morgan PJ, Plotnikoff RC, Dally KA, Salmon J, et al. Smart-phone obesity prevention trial for adolescent boys in low-income communities: the ATLAS RCT. Pediatrics. 2014;134:e723-31.

30. Lubans DR, Smith JJ, Plotnikoff RC, Dally KA, Okely AD, Salmon J, et al. Assessing the sustained impact of a school-based obesity prevention 
program for adolescent boys: the ATLAS cluster randomized controlled trial. Int J Behav NutrPhys Act. 2016;13:92.

31. Partridge SR, McGeechan K, Hebden L, Balestracci K, Wong ATY, Denney-Wilson $\mathrm{E}$, et al. Effectiveness of a mHealth lifestyle program with telephone support (TXT2BFiT) to prevent unhealthy weight gain in young adults: randomized controlled trial. JMIR Mhealth Uhealth. 2015;3:e66.

32. Allman-Farinelli M, Partridge SR, McGeechan K, Balestracci $K$, Hebden L, Wong A, et al. A mobile health lifestyle program for prevention of weight gain in young adults (TXT2BFiT): Nine-month outcomes of a randomized controlled trial. JMIR Mhealth Uhealth. 2016;4:e78.

33. Silveira $P$, van de Langenberg $R$, van het Reve E, Daniel F, Casati F, de Bruin ED. Tablet-based strength-balance training to motivate and improve adherence to exercise in independently living older people: a phase II preclinical exploratory trial. JMIR. 2013;15:114-30.

34. van het Reve E, Silveira P, Daniel F, Casati F, de Bruin ED. Tablet-based strength-balance training to motivate and improve adherence to exercise in independently living older people: part 2 of a phase II preclinical exploratory trial. JMIR. 2014;16:99-116.

35. Cowdery J, Majeske P, Frank R, Brown D. Exergame apps and physical activity: the results of the ZOMBIE trial. Am J Health Educ. 2015;46:216-22.

36. Fukuoka Y, Vittinghoff E, Jong SS, Haskell W. Innovation to motivation: Pilot study of a mobile phone intervention to increase physical activity among sedentary women. Prev Med. 2010;51:287-9.

37. Garde A, Umedaly A, Abulnaga SM, Robertson L, Junker A, Chanoine JP, et al. Assessment of a mobile game ('MobileKids Monster Manor') to promote physical activity among children. Games Health. 2015:4:145-8.

38. Gilliland J, Sadler R, Clark A, O'Connor C, Milczarek M, Doherty S. Using a smartphone application to promote healthy dietary behaviours and local food consumption. Biomed Res Int. 2015. doi:10.1155/2015/841368.

39. King AC, Hekler EB, Grieco LA, Winter SJ, Sheats JL, Buman MP, et al. Harnessing different motivational frames via mobile phones to promote daily physical activity and reduce sedentary behavior in aging adults. Plos One. 2013;8:e62613.

40. Nollen NL, Mayo MS, Carlson SE, Rapoff MA, Goggin KJ, Ellerbeck EF. Mobile technology for obesity prevention: a randomized pilot study in racial- and ethnic-minority girls. Am J Prev Med. 2014;46:404-8.

41. Stuckey M, Russell-Minda E, Read E, Munoz C, Shoemaker K, Kleinstiver P, et al. Diabetes and technology for increased activity (DaTA) study: results of a remote monitoring intervention for prevention of metabolic syndrome. J Diabetes Sci Technol. 2011;5:928-35.

42. Wharton CM, Johnston CS, Cunningham BK, Sterner D. Dietary selfmonitoring, but not dietary quality, improves with use of smartphone app technology in an 8-week weight loss trial. J Nutr Educ Behav. 2014;46:440-4.

43. Wang JB, Cadmus-Bertram LA, Natarajan L, White MM, Madanat H, Nichols JF, et al. Wearable sensor/device (Fitbit One) and SMS text-messaging prompts to increase physical activity in overweight and obese adults: a randomized controlled trial. Telemed J E Health. 2015;21:782-92.

44. Choi JW, Lee J, Vittinghoff E, Fukuoka Y. mHealth physical activity intervention: a randomized pilot study in physically inactive pregnant women. Matern Child Health. 2016;20:1091-101.

45. Mummah SA, Mathur M, King AC, Gardner CD, Sutton S. Mobile technology for vegetable consumption: a randomized controlled pilot study in overweight adults. JMIR Mhealth Uhealth. 2016;4:e51.

46. Rabbi M, Pfammatter A, Zhang M, Spring B, Choudhury T. Automated personalized feedback for physical activity and dietary behavior change with mobile phones: a randomized controlled trial on adults. JMIR Mhealth Uhealth. 2015;3:e42.

47. Direito A, Jiang Y, Whittaker R, Maddison R. Apps for IMproving FITness and increasing physical activity among young people: the AIMFIT pragmatic randomized controlled trial. JMIR. 2015;17:e210.

48. Hebden L, Cook A, van der Ploeg HP, King L, Bauman A, Allman-Farinelli M. A mobile health intervention for weight management among young adults: a pilot randomised controlled trial. J Hum Nutr Diet. 2014;27:322-32.

49. Kirwan M, Duncan MJ, Vandelanotte C, Mummery WK. Using smartphone technology to monitor physical activity in the 10,000 Steps program: a matched case-control trial. JMIR. 2012;14:e55.

50. Maher C, Ferguson M, Vandelanotte C, Plotnikoff R, De Bourdeaudhuij I, Thomas S, et al. A web-based, social networking physical activity intervention for insufficiently active adults delivered via facebook app: randomised controlled trial. JMIR. 2015;17:e174.
51. Gilson ND, Pavey TG, Vandelanotte C, Duncan MJ, Gomersall SR, Trost SG, et al. Chronic disease risks and use of a smartphone application during a physical activity and dietary intervention in Australian truck drivers. Aust N Z J Public Health. 2016:40:91-3.

52. Gasser R, Brodbeck D, Degen M, Luthiger J, Wyss R, Reichlinal S. Persuasiveness of a mobile lifestyle coaching application using social facilitation. Pers Technol. 2006;3962:27-38.

53. Glynn LG, Hayes PS, Casey M, Glynn F, Alvarez-Iglesias A, Newell J, et al. Effectiveness of a smartphone application to promote physical activity in primary care: the SMART MOVE randomised controlled trial. Br J Gen Pract. 2014;64:e384-91.

54. van Drongelen A, Boot CRL, Hlobil H, Twisk JWR, Smid T, van der Beek AJ. Evaluation of an mHealth intervention aiming to improve health-related behavior and sleep and reduce fatigue among airline pilots. Scand J Work Environ Health. 2014:40:557-68.

55. Walsh JC, Corbett T, Hogan M, Duggan J, McNamara A. An mHealth intervention using a smartphone app to increase walking behavior in young adults: a pilot study. JMIR Mhealth Uhealth. 2016;4:e109.

56. Rospo G, Valsecchi V, Bonomi AG, Thomassen IWG, van Dantzig S, La Torre A, et al. Cardiorespiratory improvements achieved by American College of sports medicine's exercise prescription implemented on a mobile app. JMIR Mhealth Uhealth. 2016;4:e77.

57. Elbert SP, Dijkstra A, Oenema A. A mobile phone app intervention targeting fruit and vegetable consumption: the efficacy of textual and auditory tailored health information tested in a randomized controlled trial. J Med Internet Res. 2016;18:e147.

58. Safran Naimark J, Madar Z, Shahar DR. The impact of a web-based app (eBalance) in promoting healthy lifestyles: randomised controlled trial. JMIR. 2015;17:e56.

59. Flecha OD, de Oliveira DWD, Marques LS, Gonçalves PF. A commentary on randomized clinical trials: How to produce them with a good level of evidence. Perspect Clin Res. 2016;7:75-80.

60. Dennison L, Morrison L, Conway G, Yardley L. Opportunities and challenges for smartphone applications in supporting health behavior change: qualitative study. JMIR. 2013;15:e86.

61. Guertler D, Vandelanotte C, Kirwan M, Duncan MJ. Engagement and nonusage attrition with a free physical activity promotion program: the case of 10,000 steps Australia. JMIR. 2015;17:e176.

62. Nikoloudakis IA, Vandelanotte C, Rebar AL, Schoeppe S, Alley S, Duncan MJ, et al. Examining the correlates of online health information-seeking behavior among men compared with women. Am J Mens Health. 2016; 2016. doi:10.1177/1557988316650625.

63. De Bourdeaudhuij I, Van Cauwenberghe E, Spittaels H, Oppert JM, Rostami C, Brug J, et al. School-based interventions promoting both physical activity and healthy eating in Europe: a systematic review within the HOPE project. Obes Rev. 2011;12:205-16.

64. Kriemler S, Meyer U, Martin E, Van Sluijs EMF, Andersen LB, Martin BW. Effect of school-based interventions on physical activity and fitness in children and adolescents: a review of reviews and systematic update. $\mathrm{Br} J$ Sports Med. 2011;45:923-30.

65. Rodrigues AL, Ball J, Ski C, Stewart S, Carrington MJ. A systematic review and meta-analysis of primary prevention programmes to improve cardiometabolic risk in non-urban communities. Prev Med. 2016;87:22-34.

66. Appleton KM, Hemingway A, Saulais L, Dinnella C, Monteleone E, Depezay L, et al. Increasing vegetable intakes: rationale and systematic review of published interventions. Eur J Nutr. 2016;2016(55):869-96.

67. Greaves CJ, Sheppard KE, Abraham C, Hardeman W, Roden M, Evans PH, et al. Systematic review of reviews of intervention components associated with increased effectiveness in dietary and physical activity interventions. BMC Public Health. 2011;11:119.

68. Vandelanotte C, Spathonis KM, Eakin EG, Owen N. Website-delivered physical activity interventions. A review of the literature. Am J Prev Med. 2007:33:54-64.

69. O'Reilly GA, Spruijt-Metz D. Current mHealth technologies for physical activity assessment and promotion. Am J Prev Med. 2013;45:501-7.

70. Pew Research Center. Teens, social media \& technology overview 2015: smartphones facilitate shifts in communication landscape for teens. Pew Research Center. 2015. http://www.pewinternet.org/files/2015/04/PI_ TeensandTech_Update2015_0409151.pdf. Accessed 5 Jul 2016.

71. Boulos MNK, Yang SP. Exergames for health and fitness: the roles of GPS and geosocial apps. Int J Health Geogr. 2013;12:18. 
72. Neve M, Morgan PJ, Jones PR, Collins CE. Effectiveness of web-based interventions in achieving weight loss and weight loss maintenance in overweight and obese adults: a systematic review with meta-analysis. Obes Rev. 2010;11:306-21.

73. Dumville JC, Torgerson DJ, Hewitt CE. Reporting attrition in randomised controlled trials. BMJ. 2006;332:969-71.

74. van Genugten L, Dusseldorp E, Webb T, van Empelen P. Which combinations of techniques and modes of delivery in internet-based interventions effectively change health behavior? A meta-analysis. JMIR. 2016;18:e155.

75. Middelweerd A, van der Laan DM, van Stralen MM, Mollee JS, Stuij M, te Velde SJ, et al. What features do Dutch university students prefer in a smartphone application for promotion of physical activity? A qualitative approach. Int J Behav Nutr Phys Act. 2015;12:31.

76. Webb TL, Joseph J, Yardley L, Michie S. Using the internet to promote health behavior change: a systematic review and meta-analysis of the impact of theoretical basis, use of behavior change techniques, and mode of delivery on efficacy. JMIR. 2010;12:e4.

77. Sandholzer M, Deutsch T, Frese T, Winter A. Predictors of students' selfreported adoption of a smartphone application for medical education in general practice. BMC Med Educ. 2015;15:91.

78. Liberati A, Altman DG, Tetzlaff J, Mulrow C, Gøtzsche PC, loannidis JP, et al. The PRISMA statement for reporting systematic reviews and meta-analyses of studies that evaluate healthcare interventions: explanation and elaboration. BMJ. 2009;339:b2700.

\section{Submit your next manuscript to BioMed Central and we will help you at every step:}

- We accept pre-submission inquiries

- Our selector tool helps you to find the most relevant journal

- We provide round the clock customer support

- Convenient online submission

- Thorough peer review

- Inclusion in PubMed and all major indexing services

- Maximum visibility for your research

Submit your manuscript at www.biomedcentral.com/submit 